\title{
Application of different imaging techniques for the characterization of damage in fiber reinforced polymer
}

\author{
Ana Vrgoč ${ }^{\mathrm{a}}$, Zvonimir Tomičević, ${ }^{\mathrm{a}, *}$, Benjamin Smaniotto ${ }^{\mathrm{b}}$, François Hild ${ }^{\mathrm{b}}$ \\ ${ }^{a}$ University of Zagreb, Faculty of Mechanical Engineering and Naval Architecture \\ Ivana Lučića 5, 10002 Zagreb, Croatia \\ ${ }^{b}$ Université Paris-Saclay, ENS Paris-Saclay, CNRS \\ LMT - Laboratoire de Mécanique et Technologie, 91190 Gif-sur-Yvette, France
}

\begin{abstract}
The heterogeneous and anisotropic nature of fiber reinforced polymers induces the initiation of various damage mechanisms at different scales. Even though full-field surface measurements may successfully track damage development, such approaches do not provide insights into the material bulk. The present work aims at comparing surface and bulk fullfield measurements (via Digital Image/Volume Correlation) for a comprehensive analysis of polymer composite damage. Bulk measurements revealed that damage initiated at the microscale, thus contributing to surface localization phenomena. Full-field surface measurements could not capture the local strain states that the constituents were subjected to, which led to final fracture.

Keywords: Digital Image Correlation (DIC), Digital Volume Correlation (DVC), Full-field measurement, Gray level residuals, Polymer matrix composite
\end{abstract}

\section{Introduction}

The increased application of lightweight structures has contributed to the development and utilization of advanced materials. Fiber reinforced polymers (FRPs) are increasingly being regarded as efficient substitutes for conventional engineering materials in critical-

\footnotetext{
${ }^{*}$ Corresponding author

Email address: zvonimir.tomicevic@fsb.hr (Zvonimir Tomičević)
} 
safety and lightweight structural applications [1]. The heterogeneous and anisotropic nature of FRPs induces the occurrence of various damage mechanisms (e.g., fiber/matrix interface debonding, matrix microcracking, fiber pull-out, fiber breakage, delamination of individual layers), which often initiate in-depth and subsequently propagate at different length scales [2]. Due to simultaneous activation of different damage mechanisms and their interactions, the failure of laminated FRPs is a direct result of events occurring at microstructural levels [3]. This strong interconnection calls for advanced techniques for early detection of both damage initiation and monitoring damage growth, which is essential to assess the state of structures and their residual strength [4].

Recent advancements in camera technology and the development of image processing algorithms have contributed to the wide application of Digital Image Correlation (DIC) in experimental mechanics [5]. The method allows in-plane displacement fields to be measured on the surface of loaded specimens. In original DIC algorithms, the acquired images were discretized into smaller zones (i.e., subsets, facets) [6, 7], and the series of subsets were registered independently. Such approaches are now referred to as local DIC [8]. In order to measure continuous displacement fields, global approaches were introduced [9], for instance based on finite element (FE) discretizations [10, 11, 12].

The utilization of DIC in experimental mechanics proved to be crucial in improving identification procedures and validations of constitutive laws [13]. Since the application of FRPs is in constant growth, extensive research has focused on identifying damage mechanisms that induce failure of engineering components. DIC has been increasingly utilized to obtain strain fields, and conduct detailed investigations of meso- and macromechanical aspects associated with FRP damage and failure [14, 15, 16, 17]. Even though such measurements efficiently detect areas of strain localization corresponding to meso- and/or macro damage locations, the influence of bulk microstructural changes on the macroscopic response was not assessed. This limitation was bypassed via coupling DIC with other NDT 
methods (such as Acoustic Emission (AE), Scanning Electron Microscopy (SEM) and Infrared Thermography (IR)) into hybrid frameworks [18, 19, 20], which enabled for more comprehensive analyses of damage extent and precise characterization of damage mechanisms. However, bulk kinematic and microstructural changes were still not assessed in situ. In this regard, Digital Volume Correlation (DVC) allows for the measurement of displacement fields and calculation of strain maps in the 3D space. DVC is the 3D volumetric extension of DIC in which the registration is performed on 3D images generally acquired with an X-ray scanner [21]. In recent years, the aforementioned approach has emerged as a powerful measurement technique in experimental solid mechanics [22, 23]. The majority of bulk damage investigations of FRP composites include applications of local DVC approaches, which enabled changes in the microstructure to be correlated with measured bulk kinematic fields, and thus to damage development in the material bulk [24, 25, 26, 27, 28]. Further, as for 2D measurements, global approaches were also introduced to register and analyze 3D volumes [29, 30]. The key advantage of global approaches is the availability of correlation residual maps, which correspond to the gray level difference between the reference volume and the volume of deformed material corrected by the measured displacement field. These residuals, which are probed for all pixels/voxels belonging to the region of interest (ROI), provide useful information when checking the convergence of DIC/DVC analyses, i.e., as the displacement fields are assumed to be continuous over the inspected ROI, the areas of elevated residuals reveal damaged zones. The analysis of correlation residual maps has proven to be successful in detecting discontinuities in both $2 \mathrm{D}$ and 3D measurements [31, 32, 33]. In addition, voxel-wise correlation residuals allowed for the detection of damage initiation and growth within the bulk of FRPs [34].

The aim of the investigation performed herein is to compare the capabilities of different image registration techniques (namely, DIC and DVC) in detecting damage onset and monitoring damage growth within a glass fiber mat reinforced polymer composite. First, a 
uniaxial and monotonic tensile experiment was conducted, and the global material behavior was assessed via FE-based DIC. The extracted major eigen strain fields and corresponding correlation residual maps are analyzed to monitor damage development on the specimen surface. Based on the reported stress-strain response, an in situ experimental study was performed to measure the 3D kinematics of the specimen subjected to cyclic tensile loading from reconstructed scans acquired with an X-ray scanner. FE-based DVC was employed to measure 3D displacements and thus calculate strain fields within the bulk of the investigated material. Before cycling the specimen, two scans were acquired in the undeformed configuration to assess measurement uncertainties. Furthermore, the first advantage of computed tomography is to reveal the underlying microstructure [35], while the calculated eigen strain fields and correlation residual maps are studied to track damage inception and growth within the loaded microstructure. To highlight the advantages of DVC compared to surface observations, a section-based DIC approach is proposed. The front and rear sections extracted from the scans acquired during cyclic tension are used as $2 \mathrm{D}$ images to perform inplane registrations. The 2D-DIC fields on the front and rear specimen surfaces are analyzed and compared to DVC results.

\section{Materials and methods}

The present section introduces the material and methods employed in the investigation performed herein. The manufacturing procedure and properties of the investigated material are presented, then followed by a brief overview of regularized DIC/DVC.

\subsection{Glass fiber mat reinforced epoxy resin composite}

The material studied herein is a continuous glass fiber mat reinforced epoxy resin composite produced by manual lay-up followed by compression molding. The investigated laminate comprised 12 plies of R-glass fiber mat laid in $438 \mathrm{~g}$ of epoxy resin mixture (LG 285 laminating resin, GRM Systems, Germany), and was cured under pressure for $24 \mathrm{~h}$ at room 
temperature. The post-curing was conducted at $100^{\circ} \mathrm{C}$ for $3 \mathrm{~h}$. As vacuum was not applied during molding, the micrographs shown in Fig. 1 reveal the presence of air voids, the volume fraction of which was found to be $5 \%$. The fiber volume fraction was $40 \%$, while that of the matrix was equal to $55 \%$. In addition, the mean fiber diameter was equal to $9 \mu \mathrm{m}$.

\section{Digital Image/Volume Correlation}

Digital Image/Volume Correlation is a widely employed technique enabling for fullfield displacement measurements [36, 37]. The general concept relies on pattern matching to measure displacement fields by correlating the image of the reference configuration $f(\mathbf{x})$ and another one of the deformed configuration $g(\mathbf{x})$. The images are $2 \mathrm{D}$ or 3D matrices of gray levels, where $\mathbf{x}$ denotes the pixel/voxel location. Gray level conservation is usually assumed to relate the two registered images

$$
f(\mathbf{x})=g(\mathbf{x}+\mathbf{u}(\mathbf{x}))
$$

where $\mathbf{u}$ is the sought displacement field. The global approaches employed herein consist in

minimizing the sum of squared differences over the ROI with respect to the sought displacement amplitudes [8]

$$
\Phi_{c}^{2}=\sum_{\mathrm{ROI}} \varphi_{c}^{2}(\mathbf{x})
$$

where $\varphi_{c}$ defines the correlation residual field

$$
\varphi_{c}(\mathbf{x})=f(\mathbf{x})-g(\mathbf{x}+\mathbf{u}(\mathbf{x})) .
$$


The objective of global DIC/DVC is to circumvent the ill-posedness of registration, and thus to reduce the measurement uncertainties by assuming continuity of the displacement field

$$
\mathbf{u}(\mathbf{x})=\sum_{n} u_{n} \boldsymbol{\Psi}_{n}(\mathbf{x})
$$

where $\boldsymbol{\Psi}_{n}$ are the selected shape functions associated with the finite element discretization of the displacement field, $u_{n}$ the associated nodal displacements. The correlation procedure consists in minimizing $\Phi_{c}^{2}$ with respect to all unknown degrees of freedom $u_{n}$, which are gathered in the column vector $\{\mathbf{u}\}$. A Gauss-Newton scheme is employed and consists in iteratively solving linear systems to determine nodal displacement corrections $\{\delta \mathbf{u}\}$

$$
[\mathbf{M}]\{\delta \mathbf{u}\}=\left\{\mathbf{b}^{\mathbf{i}}\right\}
$$

where $[\mathbf{M}]$ is the Hessian matrix comprising of shape functions $\boldsymbol{\Psi}_{\mathbf{n}}$ and image gradient $\boldsymbol{\nabla} f(\mathbf{x})$, and thus computed once for all

$$
M_{m n}=\sum_{\text {ROI }}\left(\boldsymbol{\nabla} f(\mathbf{x}) \cdot \Psi_{m}(\mathbf{x})\right)\left(\boldsymbol{\nabla} f(\mathbf{x}) \cdot \Psi_{n}(\mathbf{x})\right)
$$

$\left\{\mathbf{b}^{i}\right\}$ the residual vector at iteration $i$ accounting for the current image residuals

$$
b_{m}^{i}=\sum_{\mathrm{ROI}}\left(f(\mathbf{x})-\widetilde{g}^{i}(\mathbf{x})\right)\left(\boldsymbol{\nabla} f(\mathbf{x}) \cdot \boldsymbol{\Psi}_{m}(\mathbf{x})\right)
$$

and $\widetilde{g}^{i}(\mathbf{x})$ is equal to $g\left(\mathbf{x}+\mathbf{u}^{i}(\mathbf{x})\right)$, which is referred to as the corrected deformed image by the current estimate $\mathbf{u}^{i}$ of the displacement field.

Moreover, mechanical regularization based on the equilibrium gap in linear elasticity may be utilized to further bypass the ill-posedness of registrations, thus reducing measurement uncertainties [38, 39]. Such an approach consists in minimizing the $L_{2}$ norm of the un- 
balanced nodal forces for bulk degrees of freedom (DOFs) and traction-free surfaces (Neumann DOFs) [40]

$$
\Phi_{m}(\{\mathbf{u}\})=\{\mathbf{u}\}^{\top}\left[\mathbf{K}_{m}\right]^{\top}\left[\mathbf{K}_{m}\right]\{\mathbf{u}\}
$$

where $\Phi_{m}$ is the equilibrium gap cost function, and $\left[\mathbf{K}_{m}\right]$ the partial stiffness matrix for the aforementioned DOFs. This premise is not valid for the remaining (Dirichlet) DOFs since the external forces are not known [38, 40]. Hence, an additional penalty term is applied for the loaded boundary nodes

$$
\Phi_{S}(\{\mathbf{u}\})=\{\mathbf{u}\}^{\top}\left[\mathbf{K}_{S}\right]^{\top}[\mathbf{L}]\left[\mathbf{K}_{S}\right]\{\mathbf{u}\}
$$

where $\left[\mathbf{K}_{S}\right]$ corresponds to the partial stiffness matrix for Dirichlet nodes, and $[\mathbf{L}]$ the discrete Laplace-Beltrami operator. Thus, the total cost function $\Phi_{t}$ consists of a linear combination of DIC/DVC cost function $\Phi_{c}$, equilibrium gap $\Phi_{m}$, and penalty term for the gradient of normal tractions $\Phi_{S}$

$$
\Phi_{t}(\{\mathbf{u}\})=\frac{\omega_{c}}{\omega_{t}} \widehat{\Phi}_{c}(\{\mathbf{u}\})+\frac{\omega_{m}}{\omega_{t}} \widehat{\Phi}_{m}(\{\mathbf{u}\})+\frac{\omega_{S}}{\omega_{t}} \widehat{\Phi}_{S}(\{\mathbf{u}\}),
$$

with

$$
\begin{aligned}
& \widehat{\Phi}_{c}(\{\mathbf{u}\})=\Phi_{c}(\{\mathbf{u}\}) /\left(\{\mathbf{v}\}^{\top}[\mathbf{M}]\{\mathbf{v}\}\right), \\
& \widehat{\Phi}_{m}(\{\mathbf{u}\})=\Phi_{m}(\{\mathbf{u}\}) / \Phi_{m}(\{\mathbf{v}\}) \\
& \widehat{\Phi}_{S}(\{\mathbf{u}\})=\Phi_{S}(\{\mathbf{u}\}) / \Phi_{S}(\{\mathbf{v}\})
\end{aligned}
$$

and $\{\mathbf{v}\}$ are the nodal displacements associated with a trial displacement field corresponding to a shear wave of wavevector $\mathbf{k}$ [40], and the weighting terms are provided based on 
corresponding characteristic lengths $\xi$

$$
\omega_{c}=1, \quad \omega_{m}=\left(\xi_{m}|\mathbf{k}|\right)^{4}, \quad \omega_{S}=\left(\xi_{S}|\mathbf{k}|\right)^{4}
$$

such that

$$
\omega_{t}=\omega_{c}+\omega_{m}+\omega_{S}
$$

Mechanical regularization acts as a low-pass filter that enforces the smoothness of measured fields by means of characteristic lengths $\xi$, while leaving the low frequency resultant forces intact. When FE discretizations are employed, the regularization is activated for any $\xi$ greater than the characteristic element length, thus enhancing the convergence rate of the correlation algorithm.

One major advantage of global DIC/DVC is the availability of gray level residual $\varphi_{c}$ maps for each analyzed image/scan, which are usually not computed in local registrations [36]. Besides serving as a tool for correlation quality inspection, they provide meaningful information on the gray level conservation since the latter is deteriorated when damage occurs. Hence, in the present case, any significant local deviation to the gray level conservation or incompleteness of kinematic bases is expected to be related to damage initiation and growth.

\section{Experimental analyses}

This section addresses the experimental protocols employed in the present study. The experimental data were the applied force measured by the load cell of the testing machines and image series of specimen surface captured during the monotonic uniaxial tensile test, as well as CT scans acquired during the in situ cyclic experiment. 


\subsection{Monotonic tensile test}

Fig. 2(a) shows a $5.8 \mathrm{~mm}$ thick dogbone specimen thinned in the central part with a radius of $49 \mathrm{~mm}$ to ensure that it breaks in the ligament area. The smallest cross-section of the specimen was equal to $5.8 \times 5.8 \mathrm{~mm}^{2}$. The specimen was subjected to a displacementcontrolled monotonic and uniaxial tensile loading with a prescribed stroke rate of $2 \mathrm{~mm} / \mathrm{min}$. The ROI shown in Figure Fig. 2(a) was monitored with a single CCD camera (images were acquired at a frame rate of $3 \mathrm{fps}$ ). Three hundred thirteen images were captured; the first 50 images corresponding to the reference configuration to evaluate measurement uncertainties. An artificial black and white speckle pattern was applied onto the monitored surface to enable for displacement measurements via DIC. Table 1 gathers the hardware parameters of the optical setup.

The acquired images were analyzed via regularized FE-based DIC implemented within the Correli 3.0 framework [41]. Table 2 reports the parameters employed in the correlation analysis. In the present study, the displacement field was discretized with triangular (T3) elements with linear shape functions to properly describe the specimen boundaries. The finite element mesh employed in the DIC analysis, which was tailored to the actual specimen geometry, is shown in Fig. 2(a), The average element length, which was calculated as the square root of the mean element area, was equal to $20 \mathrm{px}$. Despite the fact that even finer FE meshes would better capture the surface kinematics (especially when the discontinuities are expected to occur), decreasing the element size yields higher measurement uncertainties. Thus, a compromise between measurement uncertainties and spatial resolution needs to be reached. The former can be further reduced by employing mechanical regularization while maintaining the same spatial resolution. However, in the present study, the regularization lengths were kept of the order of the characteristic element length (Table 2). This choice allows for heterogeneities induced by the underlying random mesostructure and localized phenomena to be more apparent in strain fields since discontinuities in displacement fields 
are less smeared out.

Prior to performing surface measurements, the displacement and strain uncertainties were evaluated with the first 50 images acquired in the unloaded configuration (Table 3). The displacement uncertainties lie in cpx range, while the corresponding level related to the maximum eigen strain is equal to $8 \times 10^{-5}$, which represents lower bounds with the selected procedure. Even though mechanical regularization was limited and the supporting FE mesh consisted of small elements, the measurement uncertainties were deemed sufficiently low.

\subsection{In situ cyclic test}

Another $5.6 \mathrm{~mm}$ thick specimen (Fig. 2(b)) was subjected to in situ cyclic tension using the TTC Deben testing machine, which enables for tension, compression and torsional loadings (Fig. 3(b) $)$. The in situ experiment was conducted in a displacement controlled mode with a velocity of $3 \mu \mathrm{m} / \mathrm{s}$. The specimen was cycled with maximum load levels corresponding to $30 \%, 60 \%$ and $90 \%$ of its ultimate tensile strength $($ Fig. 3(a) $)$. Since the proposed loading machine excludes the possibility of using the tomograph turntable, two rotational actuators allow for full rotation of the specimen during loading.

Imaging was performed on X50+ North Star Imaging tomograph of LMT. During the in situ test, 10 scans in total were acquired in loaded and unloaded stages; the first being acquired in the unloaded configuration for uncertainty quantification purposes, while the last two are scans of the fractured specimen (Fig. 3(a) $)$. The second scan was performed in a pre-loaded state (i.e., $F=400 \mathrm{~N}$ ). Two acquisition parameters (i.e., HQ-high quality and LQ-low quality) were utilized (Table 4). The HQ settings led to a scanning duration of approximately $2 \mathrm{~h}$, while the LQ settings resulted in 4 min acquisitions, thus reducing the duration of the mechanical test and mitigating relaxation phenomena. Sections of the reference volumes acquired with HQ and LQ settings are displayed in Fig. 4. The contrast between the matrix, fibers and voids allows the bulk kinematics to be measured between the various constituents. The HQ settings resulted in lower noise levels and enabled microstruc- 
tural features to be imaged in more detail. In addition, such quality was compatible with the extraction of realistic microscale meshes explicitly accounting for the morphology of the investigated composite. All the volumes were reconstructed by using a filtered-backprojection algorithm and encoded as 8-bit deep gray levels and no filtering was applied to the reconstructed volumes (Table 4). The physical size of one voxel was $14.6 \mu \mathrm{m}$, which is close to the pixel resolution used in the first experiment (Table 4). The size of the ROI was adjusted to the specimen ligament (i.e., the thinned part of the dogbone specimen) and corresponded to $610 \times 410 \times 1180$ voxels.

The reconstructed volumes represented by discrete scalar matrices of gray levels determined by the microstructure absorption of X-rays were analyzed via regularized FE-based DVC implemented within the Correli 3.0 framework [41]. The latter allows 3D displacement and strain fields to be measured in the bulk of the investigated materials [42]. The ROI investigated herein was discretized with first-order tetrahedral elements (T4) as shown in Fig. 2(b) The average element length, calculated as the cube root of the mean element volume, was equal to 18 voxels. Thus, the physical size (length) of 3D elements is close to that of 2D elements employed in DIC. Table 5 reports the parameters utilized in DVC analysis. In the present case, higher regularization lengths compared to the DIC analysis were employed to obtain a strain noise floor level similar to that reported for DIC (Table 3) since the strain fields calculated via DIC/DVC are investigated to track damage growth.

Due to lower noise level and artifacts, as filtering was not applied on the reconstructed volumes, the $\mathrm{HQ}$ scan 0 was considered as the reference for the uncertainty quantification and subsequent correlation analyses. The uncertainties, which were calculated as the standard deviation of the measured displacement and strain fields, are reported in Table 6 DVC yielded higher displacement uncertainties compared to DIC, which is related to the natural contrast image compared to artificial black and white speckle patterns, as well as the tomography technique and its associated artifacts (e.g., image noise, displacement discretization, 
ring artifacts, spurious motions during scanning [23]). Additional causes are related to the second scan (LQ 00) being acquired in a pre-loaded state, as well as the fact that it was of low quality.

\section{Results}

In this section, the results of DIC/DVC analyses to track the damage development are presented. First, regularized FE-DIC was performed to estimate the global material response during the uniaxial tensile test. The mesurands in DIC/DVC analyses are displacement fields, based on which 2D/3D eigenstrain fields were calculated. The residual maps and major eigen strain fields are analyzed to track damage propagation on the surface of the investigated specimen during the monotonic, uniaxial tensile test. 3D strain maps and voxelbased residuals of the cyclic tensile test are studied to investigate damage on various scales. In addition, the so-called section DIC procedure is proposed and run to further analyze the impact of the bulk localization phenomena on the surface observations. A comparison between DVC and section DIC results in terms of strain fields in the loading direction and correlation residual maps is presented.

\subsection{DIC results}

The global material response (i.e., stress / strain history) of the monotonic tension test was determined thanks to the optical sensor positioned over the thinned part of the observed ROI displayed in Fig. 5. The uniaxial stress was determined from the ratio of force by the initial area of the ligament. Both stress data and maximum eigen strain values extracted for different images acquired during the tensile test are reported in Table 7. From the stressstrain curve, a Young's modulus of $E=6.8 \mathrm{GPa}$ was identified, while the ultimate tensile strength was equal to $\sigma_{\max }=178 \mathrm{MPa}$.

The major eigen strain fields (Fig. 6) and correlation residual maps (Fig. 7) are reported for different load levels (Table 7) to monitor damage development on the surface of the in- 
vestigated specimen. For visualization purposes, the strain fields consist of nodal values extrapolated from the supporting FE mesh. The initial major eigen strain field corresponding to Image 40 (captured in the unloaded state) is shown in Fig. 6(a), Strain fluctuations of small magnitude were induced by camera sensor noise. With the gradual increase of load levels, the major eigen strains also increased within the entire ROI (Fig. 6(b) $)$. The first strained areas were captured for a load level of $2.8 \mathrm{kN}$ still in the linear elastic regime (Fig. 6(c) $)$. These localized phenomena initiated on the edges of the specimen ligament (the blue dashed arrows depict both initiation location and growth path of detected strained areas). The macroscopic nonlinearities are still very small, which indicates the impact of specimen machining-induced damage.

As the load levels became higher, localized phenomena were more apparent, as well as additional strained areas appeared on the edges of the sample ligament (marked with green arrows in Fig. 6(d) $)$. Since the stress/strain response became nonlinear (Fig. 5), damage propagated on the specimen surface and presumably in the bulk of the material. Consequently, numerous strained bands bypassing underlying heterogeneities are observed on the specimen surface. Further, the gradual increase of loading induced increases of the strain levels within the whole ROI (Fig. 6(e)]. Even though damage was observed in multiple areas, the final crack path following two strained bands initiated on the ligament edges was revealed in the narrowest gauge section $($ Fig. 6(f) $)$. The observed path was curved, thereby revealing the impact of underlying heterogeneities.

The same pattern of both damage initiation and growth is observed when analyzing the correlation residual maps (Fig. 7). The gray level residuals were thresholded in a way that an increase of fluctuations can be visualized. In the unloaded stage, very low residuals induced by camera sensor noise were homogeneously distributed within the ROI [Fig. 7(a) $]$, implying that DIC registration was successful. With the increase of load levels, the correlation residuals gradually increased within the observed ROI (Fig. 7(b) $)$. The first disconti- 
nuities (i.e., areas of higher correlation residuals) were detected on the edges of the sample ligament (marked with blue dashed arrows in Fig. 7(c) for Image 150 corresponding to a force equal to $2.8 \mathrm{kN}$. As the load levels became higher, localized phenomena became more evident and the presence of the dominant macrocrack in the narrowest gauge section was also revealed (Fig. 7(f) $)$.

The quality of the measured kinematics was evaluated from the correlation residual maps since higher values indicate local violations to the gray level conservation. This phenomenon makes correlation residuals the ideal field to unveil the presence of cracks when

displacement continuities were assumed. The conducted analysis proves the effectiveness of the employed methodology, i.e., regularized FE-DIC, to track damage development on the surface of composite materials. However, such an approach does not enable the extent of damage processes within the material bulk to be assessed, nor does it provide the possibility for relating microstructural and kinematic changes to damage developing in the material bulk. This may be achieved via in situ XCT coupled with FE-based DVC as discussed in the next section.

\subsection{DVC results}

During the in situ cyclic test, a total amount of 10 scans was acquired (Fig. 3(a) $)$. The registration was performed between the undeformed volume (HQ scan 0) and the deformed ones (LQ scans 00-07). Fig. 8 displays 3D renderings of major eigen strain fields for different loading and unloading steps of the cyclic test. Strain fluctuations of small magnitude are observed in the reference configuration (i.e., scan 00). When observing the major eigen strain field of the first loading step, slightly elevated strains are apparent on the front surface in the narrowest gauge section. For the subsequent unloading step, the observed areas vanish, and an essentially homogeneous strain distribution occurs apart from measurement uncertainties. Several highly strained regions are first observed for scan 03 (i.e., when the specimen was loaded up to $60 \%$ of its ultimate tensile strength). These localized phenom- 
ena are established on the ligament surfaces, which again indicates the influence of sample machining. Further, the spatial distribution of major eigen strains exhibits a horizontal strained band in the narrowest gauge section on the front specimen surface (having positive $y$ normal), as well as inclined bands contouring the underlying random mesostructure. These localized phenomena again disappear after unloading. In the last step of loading, localized phenomena in the narrowest gauge section of the specimen front surface become even more dominant.

To examine the complex kinematics occurring within the material bulk, the thresholded voxel-based strain fields are analyzed and shown in Fig. 9. In the unloaded configuration, the fluctuations induced by measurement uncertainties are hardly observed with the selected range. Even in the first loading step, the formation of an inner strained band in the narrowest gauge section is detected, already indicating the path along which the final failure occurred. In the following unloading step the strain levels decrease, and the strain field exhibits distributions within the material bulk similar to scan 00 (i.e., the strained bands were no longer active). When loading the specimen up to $60 \%$ of its ultimate tensile strength, the strained bands observed in the first loading step become more apparent (the strain levels inside the bands increase), and the strain field is now more localized. Yet, the subsequent unloading step once again results in an essentially homogeneous strain field within the material bulk (apart from fluctuations induced by measurement uncertainties). During the last loading step, multiple strained bands are observed in the investigated ROI. Localized phenomena are dominant in the narrowest gauge section and surround the fully developed crack that induced specimen failure.

The analysis of the strain fields throughout the cycling history leads to the conclusion that one cannot benefit from any ex situ measurements (i.e., in the unloaded states) since most pieces of meaningful information were lost during specimen unloading. To further prove this statement, as well as to investigate damage development within the material bulk, 
3D renderings of gray level residuals for scans acquired during the cyclic experiment are analyzed in the sequel (Fig. 10). The voxel-based residual fields were thresholded in a way that all estimated levels that fall outside of the specified range originate from mechanical causes (i.e., the focus is shifted only to an absolute range from 70 to 100 gray levels). In the reference configuration, very low residuals are homogeneously distributed within the ROI. The first discontinuities are captured for scan 03 corresponding to $60 \%$ of the ultimate tensile strength. These very localized phenomena appear near the front specimen surface $(y=457$ voxels $)$ in the section normal to the positive $y$-axis $(y=453$ voxels $)$, as well as in the material bulk. Besides these highlighted regions, the residual map in the remainder of the ROI exhibits sufficiently low values indicating successfully converged DVC calculations. In the present case, damage, which remained very localized, did not bias too much the DVC results [43]. During the following unloading step, the observed localization areas disappear. If one assumes damage initiated during the previous loading stage, the subsequent unloading induced crack closure (and damage deactivation). Further, in the last loading cycle prior to specimen failure (scan 05 corresponding to $90 \%$ of the ultimate tensile strength), multiple regions of increased residuals are observed, some of which related to the same areas observed in the corresponding field of scan 03 .

For a deeper understanding of damage development in the material bulk, the correlation residuals (Fig. 11) and major eigen strain fields (Fig. 12) of scan 05 extracted for characteristic sub-regions (1, 2 and 3) are laid over the corresponding mesostructure. Since the purpose of the conducted study was to demonstrate the capabilities of bulk measurements compared to surface observations, not all damaged areas were analyzed in detail.

For subset 1 located near the specimen front surface ( $y=457$ voxels), two characteristic sections are extracted, the first section being normal to the $y$-axis ( $y=451$ voxels) and the second being normal to the $z$-axis $(z=400$ voxels) as shown in Fig. 11. Higher correlation residuals appear along the fiber longitudinal axis, as well as on the fiber-matrix interface 
(indicated with green arrows in Fig. 11 on the corresponding sections). When observing the corresponding major eigen strain distribution (Fig. 12), the same localized phenomena occur, namely, strained bands visible in $y$ - and $z$-normal sections following the fiber contour, as well as fiber-matrix interfaces (marked with green arrows in Fig. 12).

Furthermore, the correlation residuals and major eigen strains of subset 2 are analyzed in two characteristic sections, the first being normal to the $y$-axis ( $y=429$ voxels $)$ and the second being normal to the $z$-axis $(z=798$ voxels), see Fig. 11. The correlation residual map of the section normal to the $y$-axis overlaid with the corresponding mesostructure in the background reveals elevated residuals both at fiber-matrix interfaces and along the longitudinal axis of fibers. The analysis of the correlation residuals in the $z$-normal section unveils areas with higher levels on the fiber-matrix interface near an air void (marked with green arrows in Fig. 11). Such localized phenomena (i.e., inclined strained bands containing the underlying fiber architecture) are displayed in Fig. 12.

The same procedure was followed in the analysis of correlation residuals and major eigen strains in subset 3, i.e., for $y=167$ voxels and $z=828$ voxels sections. As observed in Fig. 11, areas of elevated residuals visible in the section along the $y$-normal en-

compass the fiber region passing over an air void (marked with green arrows in Fig. 11), which contributed to damage development (since the matrix-fiber bond was damaged). This phenomenon is even more apparent in the section normal to $z($ Fig. 12 $)$ and jeopardized the integrity of the investigated specimen.

\subsection{Section DIC}

To further prove the importance of DVC (bulk) measurements, a section DIC (S-DIC) approach is proposed. The correlation analyses were conducted separately for the front and rear specimen surfaces, and the corresponding sections were extracted employing the following procedure. First, a binary mask of the reference configuration volume (HQ scan 0) was created. Next, the first and last high gradient values of the mask (corresponding to the 
front and rear surfaces) were searched for through the specimen thickness (i.e., along the $y$-direction). After obtaining the maps that contained the voxels belonging to the front and rear surfaces, a plane was fitted to these maps. Based on the plane parameters (i.e., point and normal vector), oblique sections of the front and rear specimen surface were extracted (Fig. 13). To obtain similar sections of the scans acquired during the loading/unloading history, rigid body motions were determined from the available DVC kinematics and applied to correct the position of the deformed scans to coincide with the reference scan (HQ scan 0 ). Then, the previously introduced procedure was employed to extract oblique sections of the deformed volumes.

The extracted sections were used to perform regularized in-plane DIC. The front and rear section meshes consisting of 20-pixel T3 elements were tailored to the specimen ligament edges, while the spatial distribution of the nodes coincided with the surface nodes of the 3D mesh employed in the DVC correlation (Fig. 14) to maintain consistency with DVC analyses. Further, equal regularization lengths as in DVC analyses were kept (i.e., 96 px for bulk and 64 px for Dirichlet nodes). This choice also helped S-DIC analyses to converge and measurement uncertainties to be decreased. The uncertainty levels related to displacement and normal strain in the loading direction were found to be $0.14 \mathrm{px}$ and 0.002 for the front surface, and $0.12 \mathrm{px}$ and 0.002 for the rear surface. Although mechanical regularization was employed, S-DIC resulted in higher measurement uncertainties compared to DVC registration (Table 6). It is believed that a large part of these errors was induced by the procedure for extracting planar sections. Additional sources are, as for DVC, the use of low resolution scans.

Fig. 15 shows a comparison of mean strains in the loading direction $\left(\epsilon_{z z}\right)$ for different loading/unloading steps extracted from the measured kinematic fields with virtual gauges positioned over the ROI (i.e., 2D gauge in S-DIC and 3D gauge in DVC of the same size). At the macroscale, the average strains are consistent for the three gauges. It is worth re- 
membering that areas of elevated DVC residuals were observed near the front specimen surface (Fig. 10), namely, damage initiated in the bulk and propagated toward the front surface. Yet the strain levels remained close.

From the DVC mesh, two surface meshes were also extracted. The corresponding nodal strains were obtained from the DVC results for these $2 \mathrm{D}$ meshes. The mean $\epsilon_{z z}$ strains were calculated by virtual gauges positioned over the ROI and are compared to the S-DIC levels. In both cases, DVC leads to slightly higher mean levels (Fig. 16). These results show very good consistency between DVC measurements, the corresponding surface data, and S-DIC. It was noted that the average strains for scan 02 were systematically negative even though the applied force remained positive. The origin of this result could not be elucidated.

The previous statements are further proven by comparing $\epsilon_{z z}$ fields on the front and rear surfaces calculated with both S-DIC and DVC for the last scan prior to specimen failure (Fig. 17). The color bar was limited to a maximum value of 0.06 to highlight differences in strain levels. Although local zones with higher measured strains are revealed on the front surface (namely, on the ligament edges), S-DIC yields significantly lower strain fluctuations compared to DVC (Fig. 17(a) $)$. The maximum $\epsilon_{z z}$ level of scan 05 was found to be $11.3 \%$ for DVC, while S-DIC measurements resulted in a maximum level equal to $5.4 \%$. The same trend was observed for the rear surface $($ Fig. 17(b) $)$. These results underline the added value of bulk measurements, i.e., even though areas with higher strains were captured by S-DIC, the bulk kinematics, which contributed to surface observations could not be fully assessed. The strong impact of underlying heterogeneities on surface strain fields is exemplified, as well as the importance of bulk measurements when investigating damage in composite specimens.

Last, a comparison between S-DIC and DVC was also conducted in terms of correlation residuals. Areas with high levels were not observed on the rear surface. Thus, the comparison is discussed only for the front surface (Fig. 18). Elevated residuals occurred near the 
ligament edges (marked with green circles in Fig. 18). These areas correspond to damage observed in subsets 1 and 2 (Fig. 11), which initiated in the material bulk and subsequently grew toward the specimen surface. The S-DIC residual map in the remainder of the ROI displayed sufficiently low values. This is a confirmation that, despite higher measurement uncertainties, the strain fields were considered trustworthy. It is concluded that S-DIC can successfully capture damage development on the specimen surface. However, damage initiation could not be detected.

\section{Conclusion}

The present paper compared different image based registration techniques for the analysis of damage in a polymer matrix composite (i.e., Digital Image and Volume Correlation). Monotonic and cyclic uniaxial tensile tests were performed on a continuous glass fiber mat reinforced epoxy resin composite specimens. First, FE-DIC was employed to measure kinematic fields on the surface of the investigated material during monotonic tension. The analysis of correlation residual maps and major eigen strain fields successfully revealed damage inception and growth. However, such measurements did not allow for the assessment of the 3D kinematics in the material bulk.

Additional insight into underlying damage mechanisms was obtained by performing an in situ cyclic test on a dogbone specimen imaged via X-ray tomography. Bulk kinematic fields were measured via FE-DVC. The analysis of 3D major eigen strain fields revealed several strained bands not only on the surfaces but in the material bulk as well. When observing the strain fields of unloaded steps after strain localization onset, the strained bands were no longer active, and the strain levels significantly decreased. The same trend was found for the voxel-based residual fields, namely, the discontinuities observed when cycling the specimen up to $60 \%$ of its ultimate tensile strength were no longer visible. These phenomena highlight the importance of in situ measurements since ex situ analyses (i.e., 
imaging the unloaded sample) would have resulted in a loss of information beneficial for damage detection. In addition, the residual fields and major eigen strain fields laid over the corresponding mesostructure enabled damage initiation to be assessed.

In order to further highlight the advantages of bulk measurements, so-called section DIC (or S-DIC) was implemented and conducted on the front and rear surfaces of the sample cycled in tension. It was shown that S-DIC resulted in lower strain fluctuations on both surfaces, which exemplified the impact of bulk kinematic changes on surface observations. S-DIC residual maps revealed zones of higher residuals on the ligament edges of the front surface corresponding to the areas observed when analyzing DVC residual fields. The latter ones proved that damage initiated in the material bulk and subsequently propagated toward the specimen surface. Even though S-DIC efficiently revealed macrocracks on the specimen surface, the first discontinuities appeared within the material bulk and could not be assessed with such measurements. 


\section{CRediT author statement}

Ana Vrgoč: Software, Formal analysis, Writing - Original Draft, Visualization

Zvonimir Tomičević: Conceptualization, Validation, Resources, Writing - Review and

Editing, Supervision, Project administration

Benjamin Smaniotto: Investigation

François Hild: Software, Validation, Writing - Review and Editing, Supervision

\section{Compliance with Ethical Standards}

The authors have no conflict of interest to declare.

\section{Acknowledgments}

This work was conducted within the FULLINSPECT project supported by the Croatian Science Foundation (UIP-2019-04-5460 Grant). 


\section{References}

[1] Prashanth S, Km S, Nithin K, Sachhidan . Fiber reinforced composites - a review. J Material Sci Eng 2017;6:1-6.

[2] Brunner A. Identification of damage mechanisms in fiber-reinforced polymer-matrix composites with acoustic emission and the challenge of assessing structural integrity and service-life. Constr Build Mater 2018;173:629-37.

[3] Nguyen VD, Wu L, Noels L. A micro-mechanical model of reinforced polymer failure with length scale effects and predictive capabilities. validation on carbon fiber reinforced high-crosslinked rtm6 epoxy resin. Mech Mater 2019;133:193-213.

[4] Bělský P, Kadlec M. Capability of non-destructive techniques in evaluating damage to composite sandwich structures. Int J Struct Integr 2019;10:356-70.

[5] Sutton M. Computer vision-based, noncontacting deformation measurements in mechanics: A generational transformation. Appl Mech Rev 2013;65(AMR-131009):050802.

[6] Sutton M, Wolters W, Peters W, Ranson W, McNeill S. Determination of displacements using an improved digital correlation method. Im Vis Comp 1983;1(3):133-9.

[7] Chu T, Ranson W, Sutton M, Peters W. Applications of digital-image-correlation techniques to experimental mechanics. Exp Mech 1985;3(25):232-44.

[8] Hild F, Roux S. Comparison of local and global approaches to digital image correlation. Exp Mech 2012;52:1503-19.

[9] Wagne B, Roux S, Hild F. Spectral approach to displacement evaluation from image analysis. Eur Phys J AP 2002;17:247-52. 
[10] Broggiato G. Adaptive image correlation technique for full-field strain measurement. In: Pappalettere C, editor. 12th Int. Conf. Exp. Mech. McGraw Hill, Lilan (Italy); 2004, p. 420-1.

[11] Sun Y, Pang J, Wong C, Su F. Finite-element formulation for a digital image correlation method. Appl Optics 2005;44(34):7357-63.

[12] Besnard G, Hild F, Roux S. "Finite-element" displacement fields analysis from digital images: Application to Portevin-Le Chatelier bands. Exp Mech 2006;46:789-803.

[13] Grédiac M, Hild F, editors. Full-Field Measurements and Identification in Solid Mechanics. London (UK): ISTE / Wiley; 2012.

[14] Brynk T, Molak RM, Janiszewska M, Pakiela Z. Digital image correlation measurements as a tool of composites deformation description. Comput Mater Sci 2012;64:157-61.

[15] Haris A, Tay T, Tan V. Experimental analysis of composite bolted joints using digital image correlation. J Mech Eng Sci 2017;14:2443-55.

[16] Khechai A, Tati A, Guerira B, Guettala A, Mohite PM. Strength degradation and stress analysis of composite plates with circular, square and rectangular notches using digital image correlation. Compos Struct 2018;185:699-715.

[17] Szebényi G, Hliva V. Detection of delamination in polymer composites by digital image correlation—experimental test. Polymers 2019;11.

[18] Godara A, Raabe D. Influence of fiber orientation on global mechanical behavior and mesoscale strain localization in a short glass-fiber-reinforced epoxy polymer composite during tensile deformation investigated using digital image correlation. Compos Sci Technol 2007;67:2417-27. 
[19] Muc A, Barski M, Chwał M, Romanowicz P, Stawiarski A. Fatigue damage growth monitoring for composite structures with holes. Compos Struct 2018;189:117-26.

[20] Kolanu NR, Raju G, Ramji M. Damage assessment studies in cfrp composite laminate with cut-out subjected to in-plane shear loading. Compos Part B Eng 2019;166:25771.

[21] Gigliotti M, Pannier Y, Gonzalez RA, Lafarie-Frenot MC, Lomov S. X-ray microcomputed-tomography characterization of cracks induced by thermal cycling in noncrimp 3d orthogonal woven composite materials with porosity. Compos Part A Appl Sci Manuf 2018;112:100-10.

[22] Bay B. Methods and applications of digital volume correlation. J Strain Analysis 2008;43:745-60.

[23] Buljac A, Jailin C, Mendoza A, Neggers J, Taillandier-Thomas T, Bouterf A, et al. Digital volume correlation: Review of progress and challenges. Exp Mech 2018;58:661-708.

[24] Brault R, Germaneau A, Dupré J, Doumalin P, Mistou S, Fazzini M. In-situ analysis of laminated composite materials by x-ray micro-computed tomography and digital volume correlation. Exp Mech 2013;53:1143-51.

[25] Croom B, Wang WM, jing Li J, Li X. Unveiling 3d deformations in polymer composites by coupled micro x-ray computed tomography and volumetric digital image correlation. Exp Mech 2016;56:999-1016.

[26] Wang K, Pei S, Li Y, Li J, Zeng D, Su X, et al. In-situ 3d fracture propagation of short carbon fiber reinforced polymer composites. Compos Sci Technol 2019;182:107788. 
[27] Hanhan I, Agyei RF, Xiao X, Sangid M. Predicting microstructural void nucleation in discontinuous fiber composites through coupled in-situ x-ray tomography experiments and simulations. Sci Rep 2020;10.

[28] Agyei RF, Hanhan I, Sangid M. Detecting damage initiation in short fiber composites via in-situ x-ray tomography and digital volume correlation. Compos Commun $2020 ; 22: 100524$.

[29] Roux S, Hild F, Viot P, Bernard D. Three dimensional image correlation from X-Ray computed tomography of solid foam. Comp Part A 2008;39(8):1253-65.

[30] Réthoré J, Tinnes J, Roux S, Buffière J, Hild F. Extended three-dimensional digital image correlation (X3D-DIC). C R Mécanique 2008;336:643-9.

[31] Tomičević Z, Roux S, Hild F. Evaluation of fatigue crack network growth in cast iron for different biaxial loading paths via full-field measurements. Int J Fatigue 2016;92:281-303.

[32] Mazars V, Caty O, Couégnat G, Bouterf A, Roux S, Denneulin S, et al. Damage investigation and modeling of $3 \mathrm{~d}$ woven ceramic matrix composites from x-ray tomography in-situ tensile tests. Acta Mater 2017;140:130-9.

[33] Buljac A, Helfen L, Hild F, Morgeneyer T. Effect of void arrangement on ductile damage mechanisms in nodular graphite cast iron: In situ 3d measurements. Eng Fract Mech 2018;192:242-61.

[34] Tomičević Z, Bouterf A, Surma R, Hild F. Damage observation in glass fiber reinforced composites via $\mu$-tomography. Materials Today: Proceedings 2019;12:185-91.

[35] Maire E, Withers PJ. Quantitative X-ray tomography. Int Mat Rev 2014;59(1):1-43. 
[36] Sutton M, Orteu J, Schreier H. Image correlation for shape, motion and deformation measurements: Basic Concepts, Theory and Applications. New York, NY (USA): Springer; 2009.

[37] Hild F, Roux S. Digital image correlation. In: Rastogi P, Hack E, editors. Optical Methods for Solid Mechanics. A Full-Field Approach. Weinheim (Germany): WileyVCH; 2012, p. 183-228.

[38] Tomičević Z, Hild F, Roux S. Mechanics-aided digital image correlation. J Strain Anal Eng Des 2013;48:330 -43.

[39] Leclerc H, Périé J, Hild F, Roux S. Digital volume correlation: What are the limits to the spatial resolution? Mech \& Indust 2012;13:361-71.

[40] Mendoza A, Neggers J, Hild F, Roux S. Complete mechanical regularization applied to digital image and volume correlation. Comput Method Appl M 2019;355:27-43.

[41] Leclerc H, Neggers J, Mathieu F, Hild F, Roux S. Correli 3.0. IDDN.FR.001.520008.000.S.P.2015.000.31500; Agence pour la Protection des Programmes, Paris (France); 2015.

[42] Hild F, Bouterf A, Chamoin L, Mathieu F, Neggers J, Pled F, et al. Toward 4d mechanical correlation. Adv Mech Simul Eng Sci 2016;3(1):1-26.

[43] Croom B, Jin H, Mills B, Li X. Effect of fragile speckle patterns on accuracy of digital volume correlation. Exp Mech 2019;59:991-1005. 


\section{List of Figures}

1 Cross sections of the composite microstructure at two different magnifications obtained by optical microscopy. . . . . . . . . . . . . . . . 32

2 Geometries of investigated dogbone specimens together with (a) 20-pixel triangular elements employed in DIC analyses, and (b) 18-voxel tetrahedral elements utilized in DVC registrations. The red contours depict the regions of interest. The size of the region of interest employed in DIC was $2040 \times$ 615 pixels, and the physical length of one pixel corresponded to $16 \mu \mathrm{m}$. The size of the region of interest considered in DVC was $610 \times 410 \times 1180$ voxels, and the physical length of one voxel was equal to $14.6 \mu \mathrm{m}$

3 (a) Schematic representation of the loading and acquisition history for the in situ tensile test. LQ and HQ symbols denote the positions at which a new scan was acquired. The first acquisition was performed in the unloaded state and was considered as the reference to assess measurement uncertainties and run correlation analyses. Then, eight additional scans were acquired for the in situ bulk kinematic measurements. One post-mortem HQ scan was finally performed. (b) Deben testing machine. . . . . . . . . . . . . . . . . . 34

4 Orthoslices of the reference volumes acquired with (a) high quality (HQ) and (b) low quality (LQ) scanning settings corresponding to scans 0 and 00 , respectively (Fig. 3). The red contours depict the region of interest whose size was $610 \times 410 \times 1180$ voxels. The physical length of one voxel was

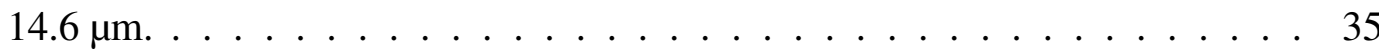


5 Stress / strain response of the monotonic experiment. The solid black circles denote the images for which the correlation residual maps and major eigen strain fields are analyzed to detect damage. The blue rectangle plotted over the major eigen strain field calculated prior to the specimen failure depicts the virtual gauge used to calculate the mean strains in the thinned part of the investigated region of interest. . . . . . . . . . . . 36

6 Major eigen strain fields for different load levels of the monotonic tensile test. The blue dashed arrows depict the initiation location and growth path of the first detected damage (i.e., zones in which eigen strains increase with prescribed load), while the green dashed arrows indicate additional areas of elevated major eigen strains. The size of the region of interest corresponded

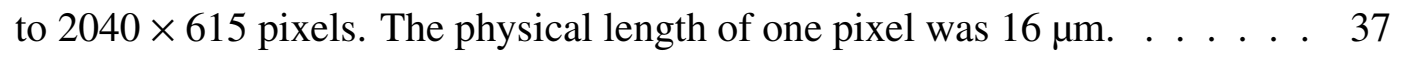

7 Correlation residual maps for different load levels of the monotonic tensile test. The blue dashed arrows depict initiation locations and growth path of the first detected discontinuities (i.e., zones in which residuals increase with prescribed load), while the green dashed arrows indicate the additional areas of higher correlation residuals. The size of the region of interest was equal

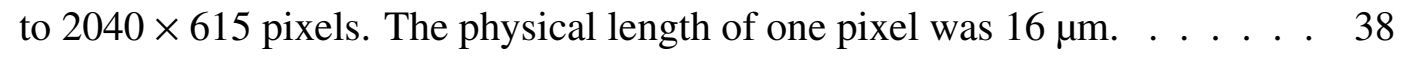

$8 \quad 3 \mathrm{D}$ renderings of the major eigen strain fields captured in the undeformed configuration (scan 00) and subsequent loading/unloading steps (Fig. 3(a)). The size of the region of interest was equal to $610 \times 410 \times 1180$ voxels, and

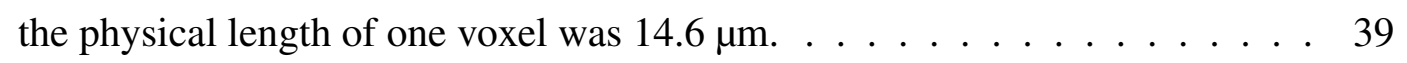

$9 \quad$ Thresholded major eigen strain fields calculated for the undeformed configuration (scan 00) and subsequent loading/unloading steps (Fig. 3(a)). The size of the region of interest corresponded to $610 \times 410 \times 1180$ voxels. The

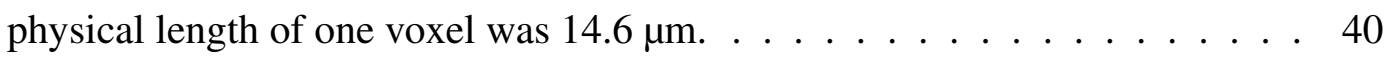


10 Isometric view of the thresholded gray level residual fields for the undeformed configuration (scan 00) and subsequent loading and unloading steps (Fig. 3(a)). The size of the region of interest was $610 \times 410 \times 1180$ voxels, and the physical length of one voxel was $14.6 \mu \mathrm{m}$. . . . . . . . . . . . . . . . 41

11 Correlation residuals (scan 05) laid over the corresponding mesostructure sections. The subsets for which comprehensive analyses of correlation residuals and major eigen strain fields are performed are denoted as 1,2 and 3. The green arrows indicate areas of increased correlation residuals in two perpendicular planes. . . . . . . . . . . . . . . . . . . . . . 42

12 Major eigen strain fields (scan 05) plotted over the corresponding mesostructure sections. Characteristics subsets for which comprehensive analyses of correlation residuals and major eigen strain fields are conducted are denoted with 1,2 and 3. The green arrows indicate strain localization areas in two perpendicular planes corresponding to regions of increased correlation residuals, as noted in|Fig. 11. . . . . . . . . . . . . . . . . . 43

13 2D front and rear sections of HQ scan 0 (Fig. 3(a)). The corresponding front and rear sections of each acquired scan are then used to perform S-DIC. . . . 44

$14 \quad$ 2D mesh employed in S-DIC procedure (red) and DVC mesh (blue) plotted over the corresponding mesostructure for the front (a) and rear (b) specimen surfaces. . . . . . . . . . . . . . . . . . . . 45

15 Comparison of mean $\epsilon_{z z}$ strain levels extracted from DVC and S-DIC runs. The mean strain levels are calculated with 2D and 3D virtual gauges of same size positioned over the ROI. The position and size of the gauge are depicted with the blue box on the S-DIC front surface showing $\epsilon_{z z}$ strain field $(\operatorname{scan} 05) . \ldots \ldots \ldots$. . . . . . . . . . . . . . . . 46 
16 Comparison of mean $\epsilon_{z z}$ strain levels extracted from the front/rear DVC and S-DIC runs. The mean strain levels are calculated via 2D virtual gauges of same size put over the ROI. The gauge position and size are depicted with the blue box in the S-DIC front surface showing $\epsilon_{z z}$ strain field (scan 05). . . . 47

17 Comparison of longitudinal strain fields $\varepsilon_{z z}$ obtained with S-DIC (left) and DVC (right) for scan 05 on (a) the front, and (b) rear surfaces. The black arrows depict the location of maximum strain. . . . . . . . . . . . . . . . . . 48

18 Comparison of correlation residual fields on the front specimen surface obtained with S-DIC (left) and DVC (right) for scan 05. The green circles depict the areas of higher residuals observed on the specimen front surface. . . . 49 


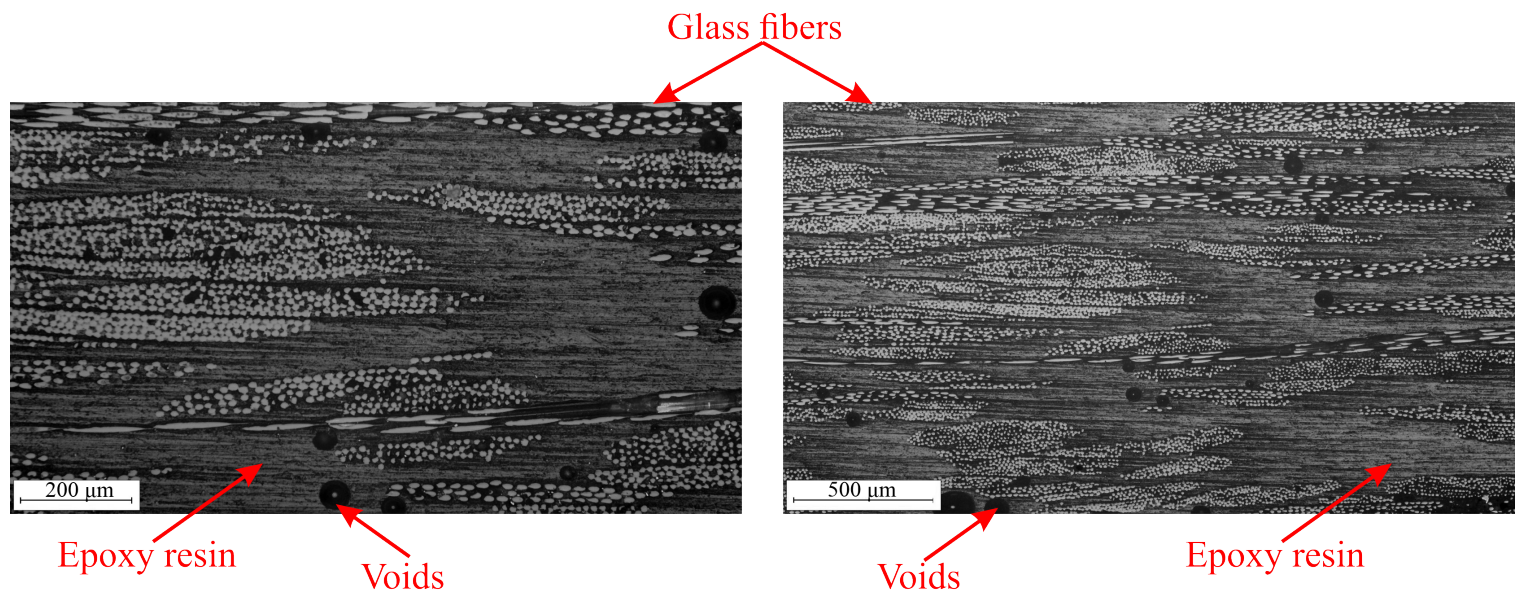

Fig 1. Cross sections of the composite microstructure at two different magnifications obtained by optical microscopy. 


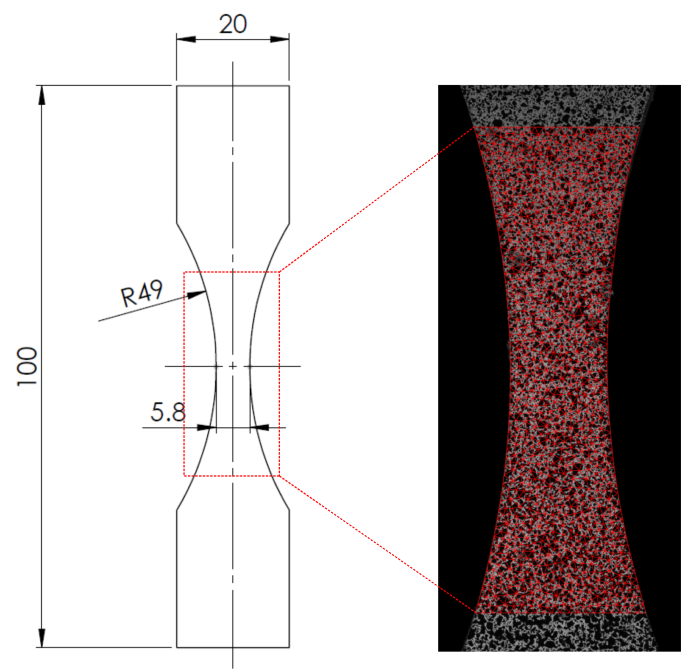

(a)

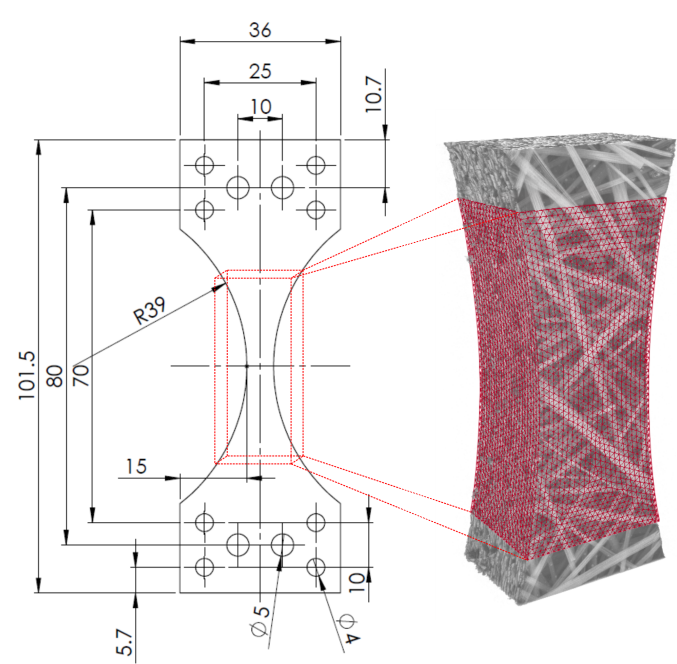

(b)

Fig 2. Geometries of investigated dogbone specimens together with (a) 20-pixel triangular elements employed in DIC analyses, and (b) 18-voxel tetrahedral elements utilized in DVC registrations. The red contours depict the regions of interest. The size of the region of interest employed in DIC was $2040 \times 615$ pixels, and the physical length of one pixel corresponded to $16 \mu \mathrm{m}$. The size of the region of interest considered in DVC was $610 \times 410 \times 1180$ voxels, and the physical length of one voxel was equal to $14.6 \mu \mathrm{m}$ 


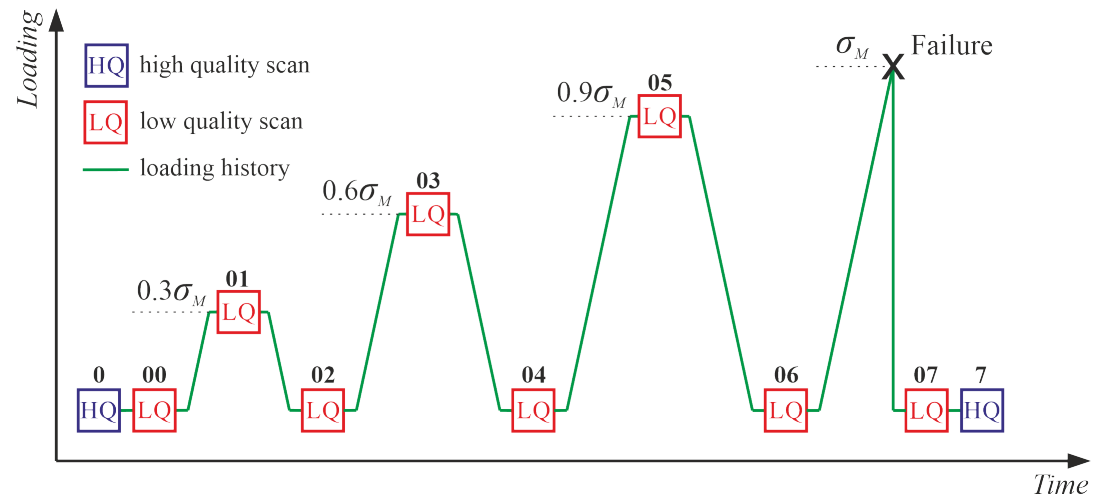

(a)

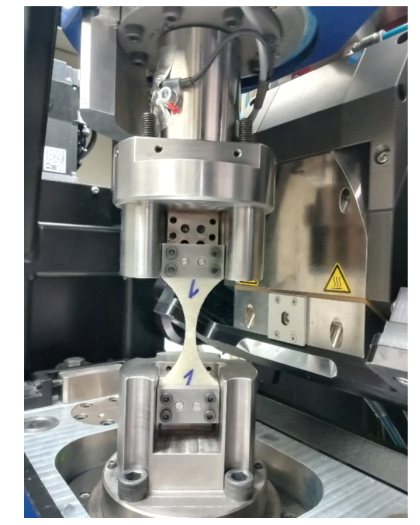

(b)

Fig 3. (a) Schematic representation of the loading and acquisition history for the in situ tensile test. LQ and HQ symbols denote the positions at which a new scan was acquired. The first acquisition was performed in the unloaded state and was considered as the reference to assess measurement uncertainties and run correlation analyses. Then, eight additional scans were acquired for the in situ bulk kinematic measurements. One postmortem HQ scan was finally performed. (b) Deben testing machine. 


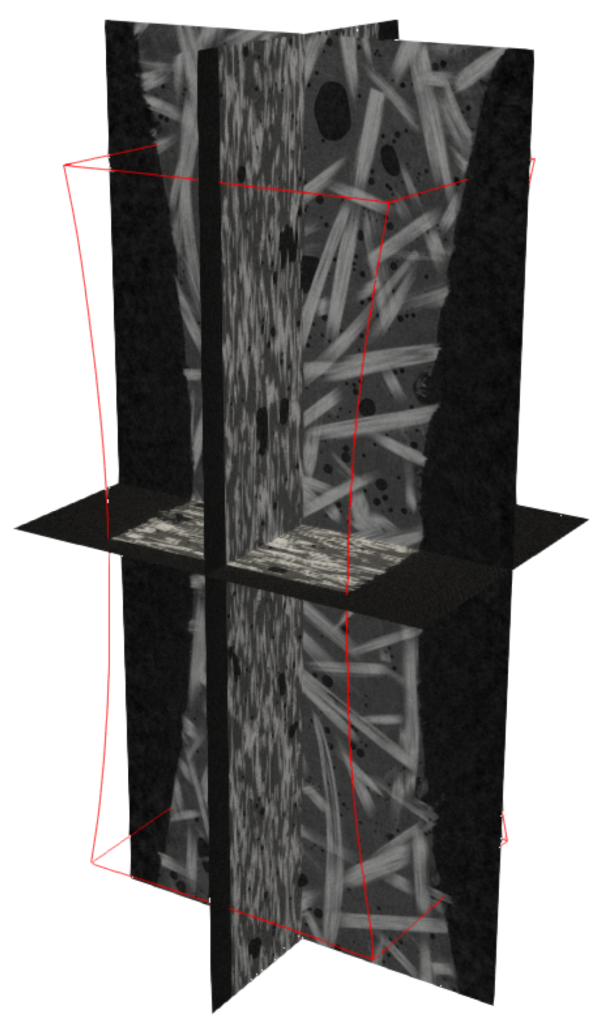

(a)

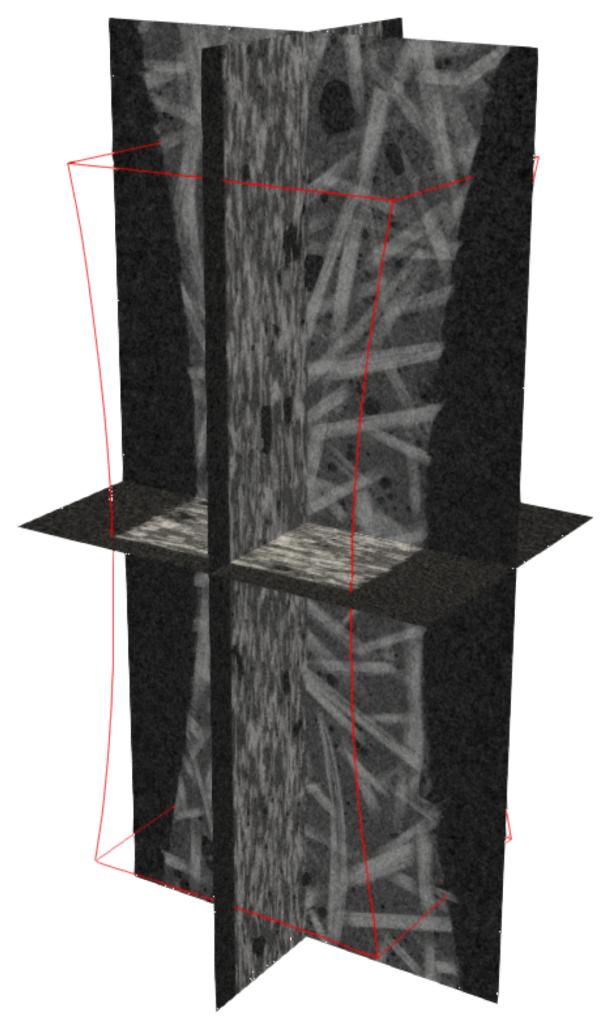

(b)

Fig 4. Orthoslices of the reference volumes acquired with (a) high quality (HQ) and (b) low quality (LQ) scanning settings corresponding to scans 0 and 00 , respectively (Fig. 3). The red contours depict the region of interest whose size was $610 \times 410 \times 1180$ voxels. The physical length of one voxel was $14.6 \mu \mathrm{m}$. 


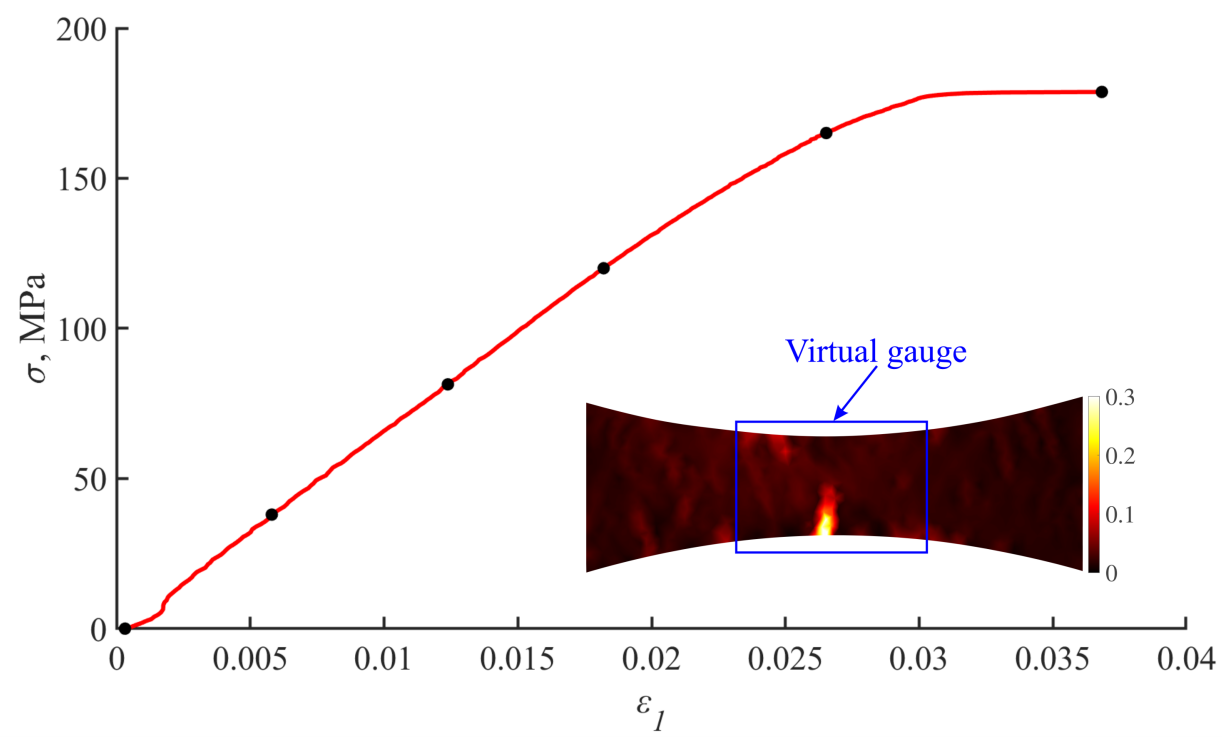

Fig 5. Stress / strain response of the monotonic experiment. The solid black circles denote the images for which the correlation residual maps and major eigen strain fields are analyzed to detect damage. The blue rectangle plotted over the major eigen strain field calculated prior to the specimen failure depicts the virtual gauge used to calculate the mean strains in the thinned part of the investigated region of interest. 


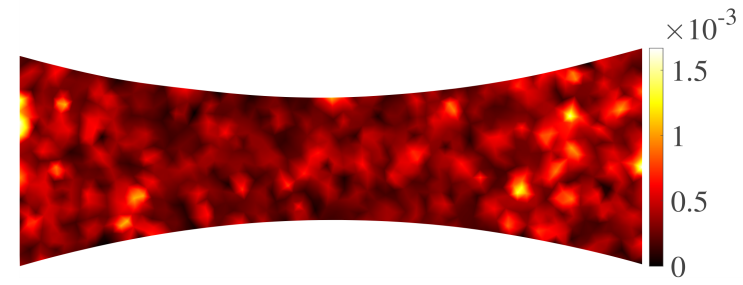

(a) $F=0 \mathrm{kN}($ Image 40)

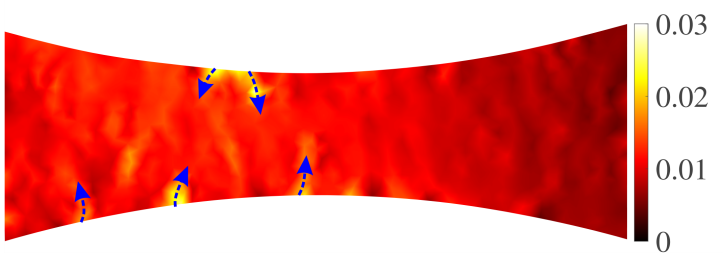

(c) $F=2.8 \mathrm{kN}($ Image 150)

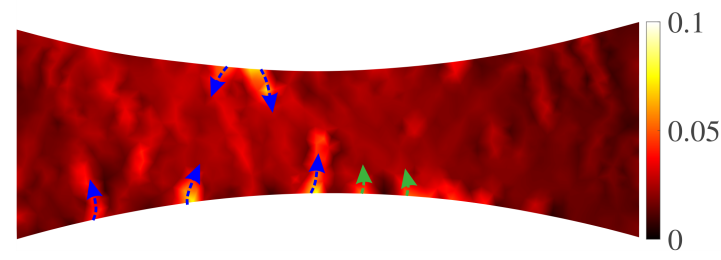

(e) $F=5.5 \mathrm{kN}($ Image 265)

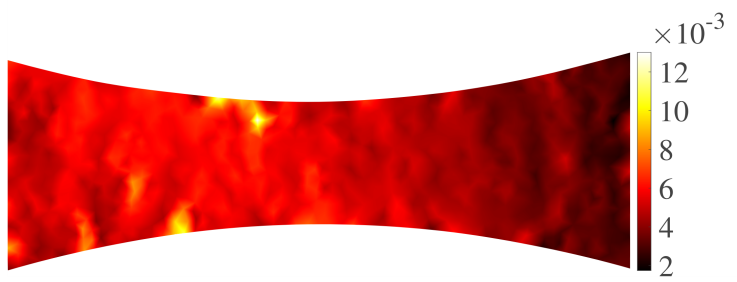

(b) $F=1.2 \mathrm{kN}($ Image 100)

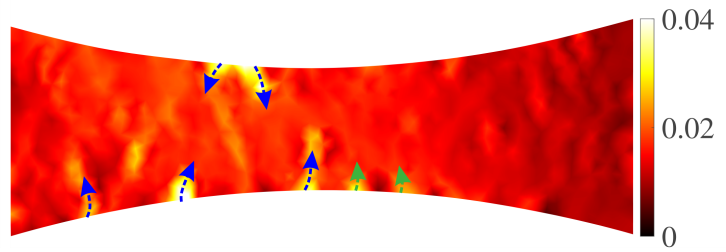

(d) $F=3.9 \mathrm{kN}($ Image 195)

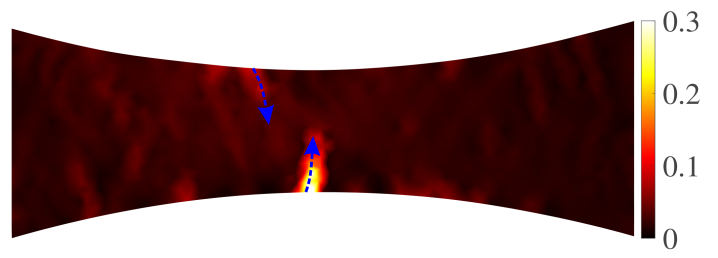

(f) $F=6.3 \mathrm{kN}($ Image 299)

Fig 6. Major eigen strain fields for different load levels of the monotonic tensile test. The blue dashed arrows depict the initiation location and growth path of the first detected damage (i.e., zones in which eigen strains increase with prescribed load), while the green dashed arrows indicate additional areas of elevated major eigen strains. The size of the region of interest corresponded to $2040 \times 615$ pixels. The physical length of one pixel was $16 \mu \mathrm{m}$. 


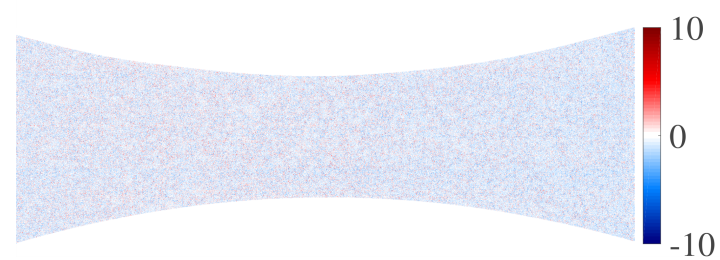

(a) $F=0 \mathrm{kN}($ Image 40)

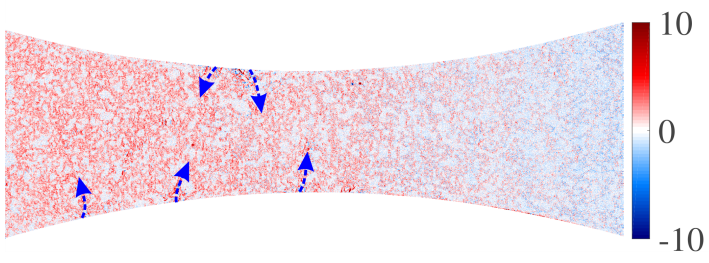

(c) $F=2.8 \mathrm{kN}($ Image 150)

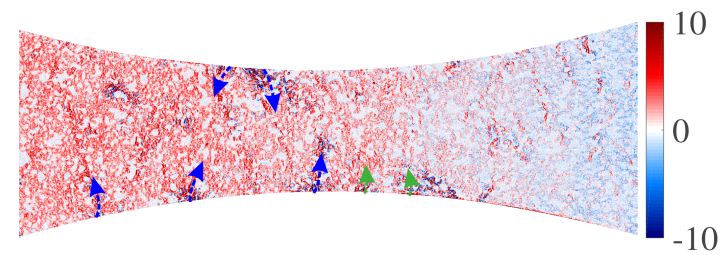

(e) $F=5.5 \mathrm{kN}($ Image 265)

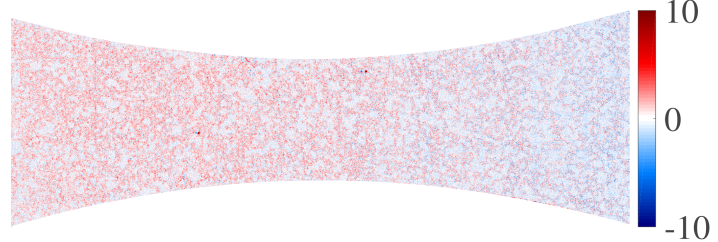

(b) $F=1.2 \mathrm{kN}($ Image 100)

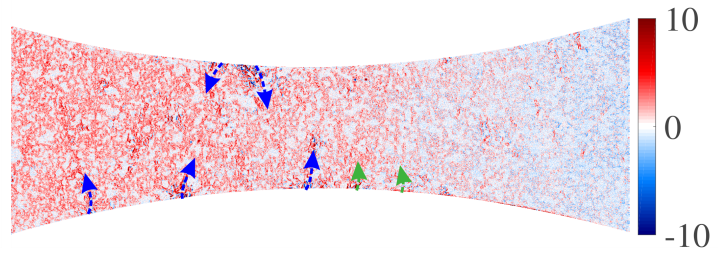

(d) $F=3.9 \mathrm{kN}($ Image 195)

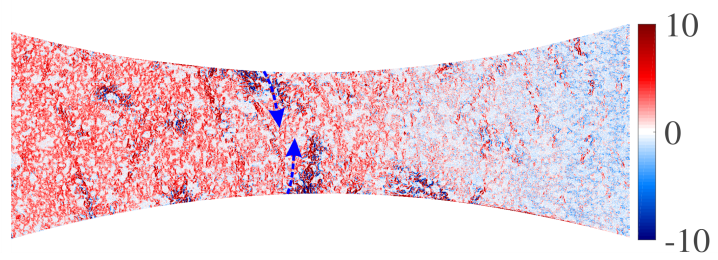

(f) $F=6.3 \mathrm{kN}($ Image 299)

Fig 7. Correlation residual maps for different load levels of the monotonic tensile test. The blue dashed arrows depict initiation locations and growth path of the first detected discontinuities (i.e., zones in which residuals increase with prescribed load), while the green dashed arrows indicate the additional areas of higher correlation residuals. The size of the region of interest was equal to $2040 \times 615$ pixels. The physical length of one pixel was $16 \mu \mathrm{m}$. 
Scan 00

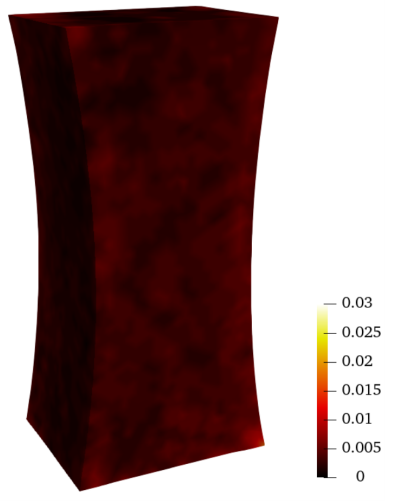

Scan 03

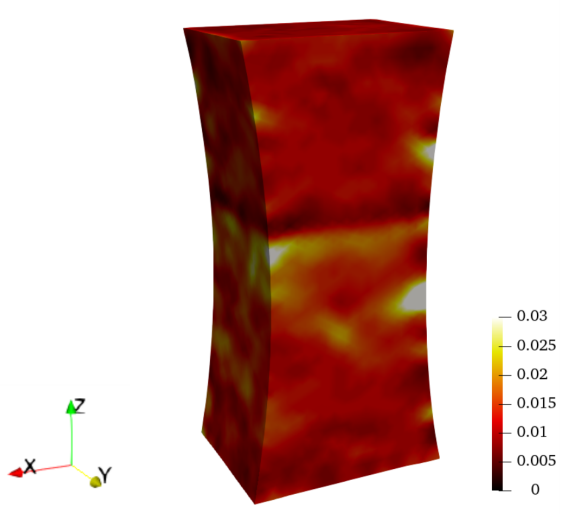

Scan 01

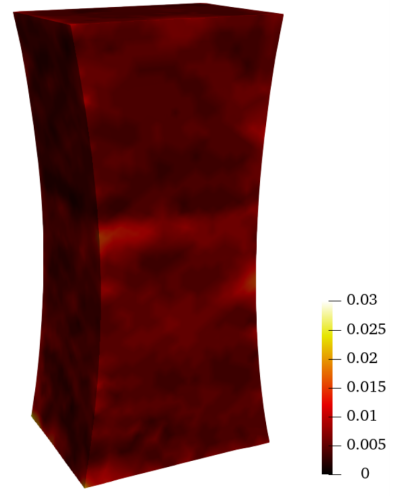

Scan 04

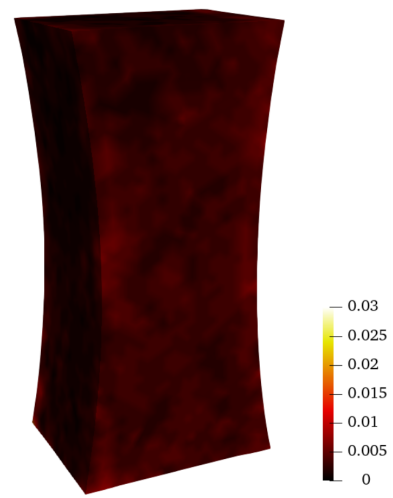

Scan 02

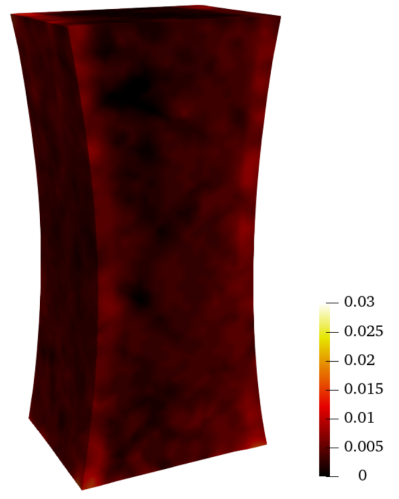

Scan 05

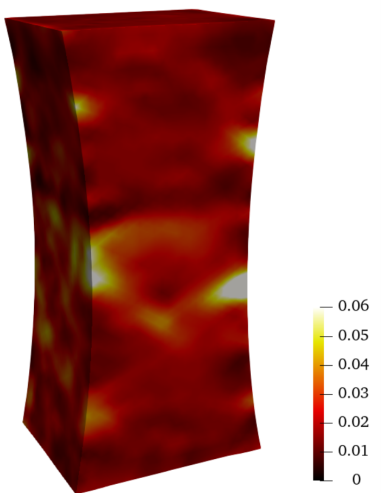

Fig 8. 3D renderings of the major eigen strain fields captured in the undeformed configuration (scan 00) and subsequent loading/unloading steps $[$ Fig. 3(a) . The size of the region of interest was equal to $610 \times 410 \times 1180$ voxels, and the physical length of one voxel was $14.6 \mu \mathrm{m}$. 
Scan 00

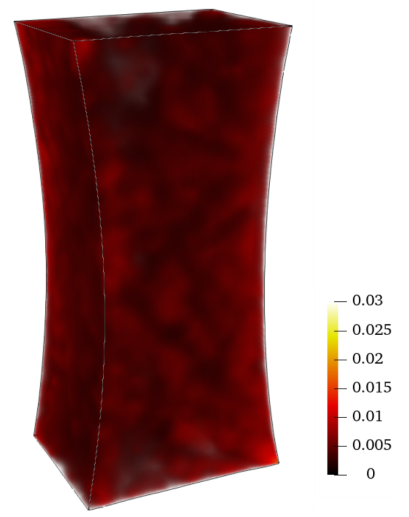

Scan 03

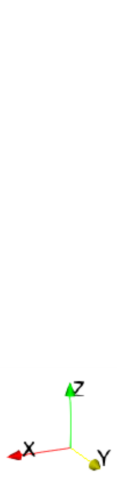

Scan 01

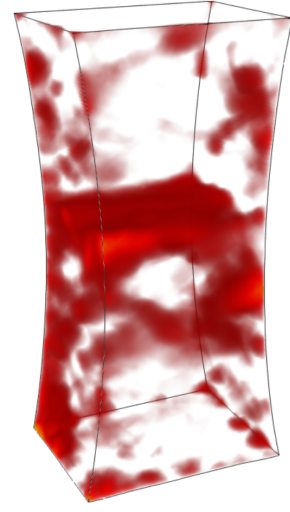

Scan 04

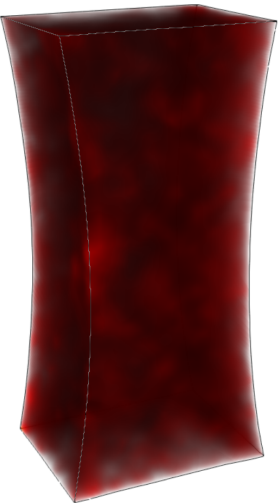

Scan 02

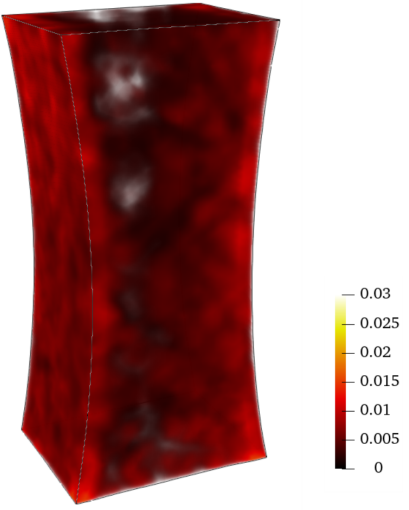

Scan 05

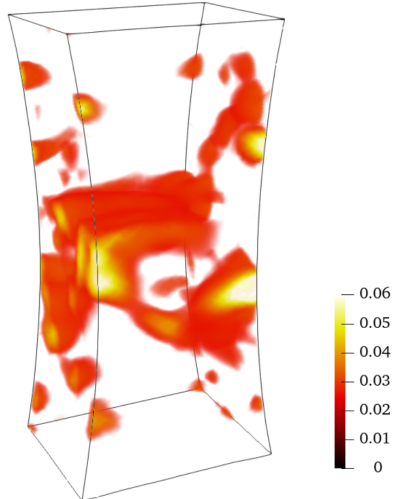

Fig 9. Thresholded major eigen strain fields calculated for the undeformed configuration (scan 00) and subsequent loading/unloading steps (Fig. 3(a) $)$. The size of the region of interest corresponded to $610 \times 410 \times 1180$ voxels. The physical length of one voxel was $14.6 \mu \mathrm{m}$. 


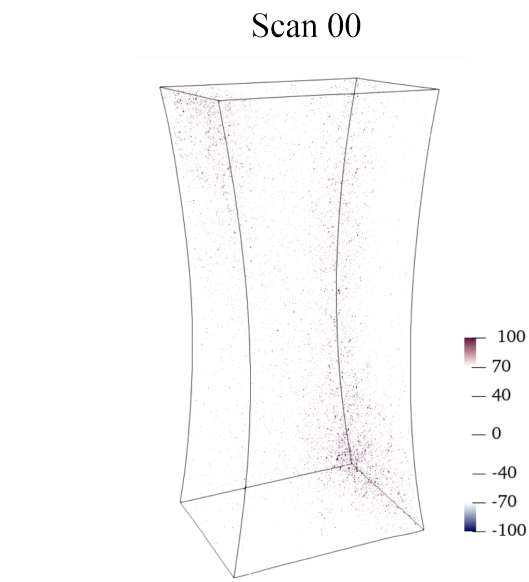

Scan 01

Scan 02

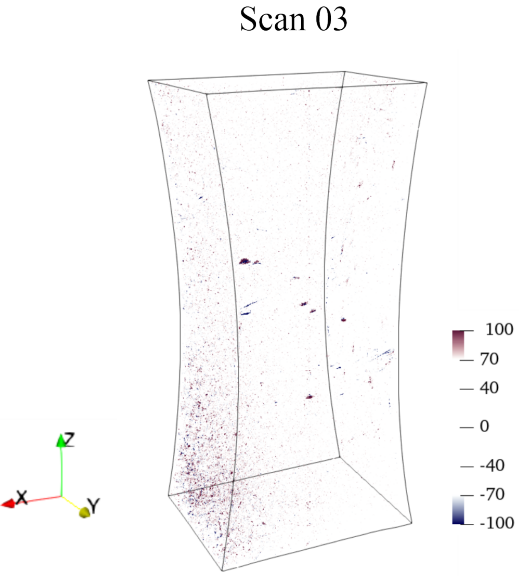

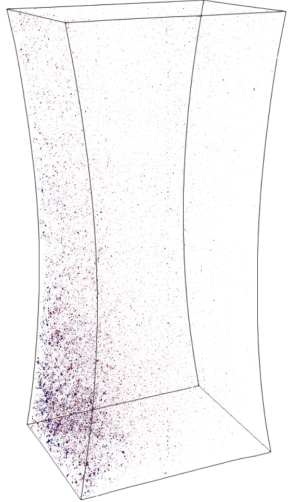

Scan 04

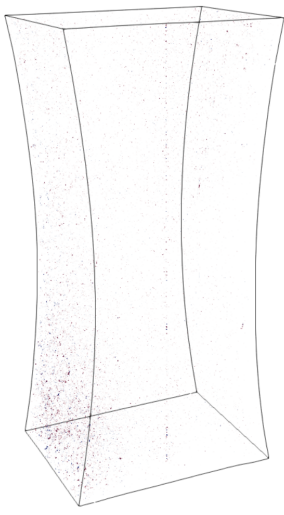

100
-70

$-40$

$-0$

$--40$

-.70
-100

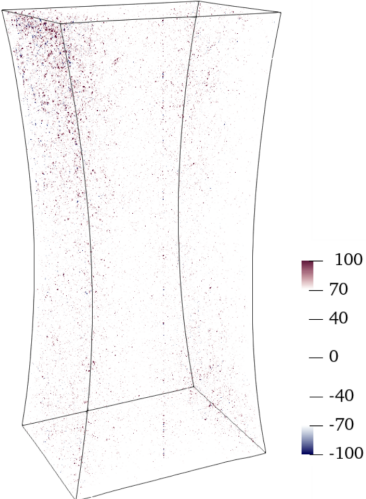

Scan 05

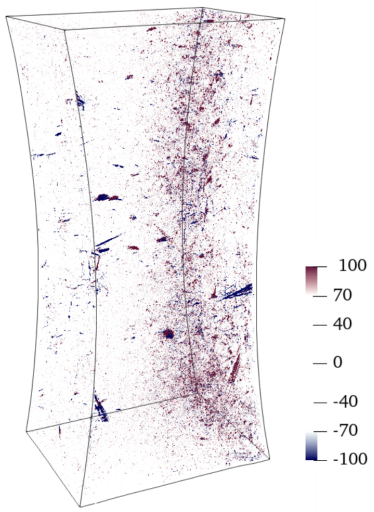

Fig 10. Isometric view of the thresholded gray level residual fields for the undeformed configuration (scan 00) and subsequent loading and unloading steps $[$ Fig. 3(a) $]$. The size of the region of interest was $610 \times 410 \times 1180$ voxels, and the physical length of one voxel was $14.6 \mu \mathrm{m}$. 


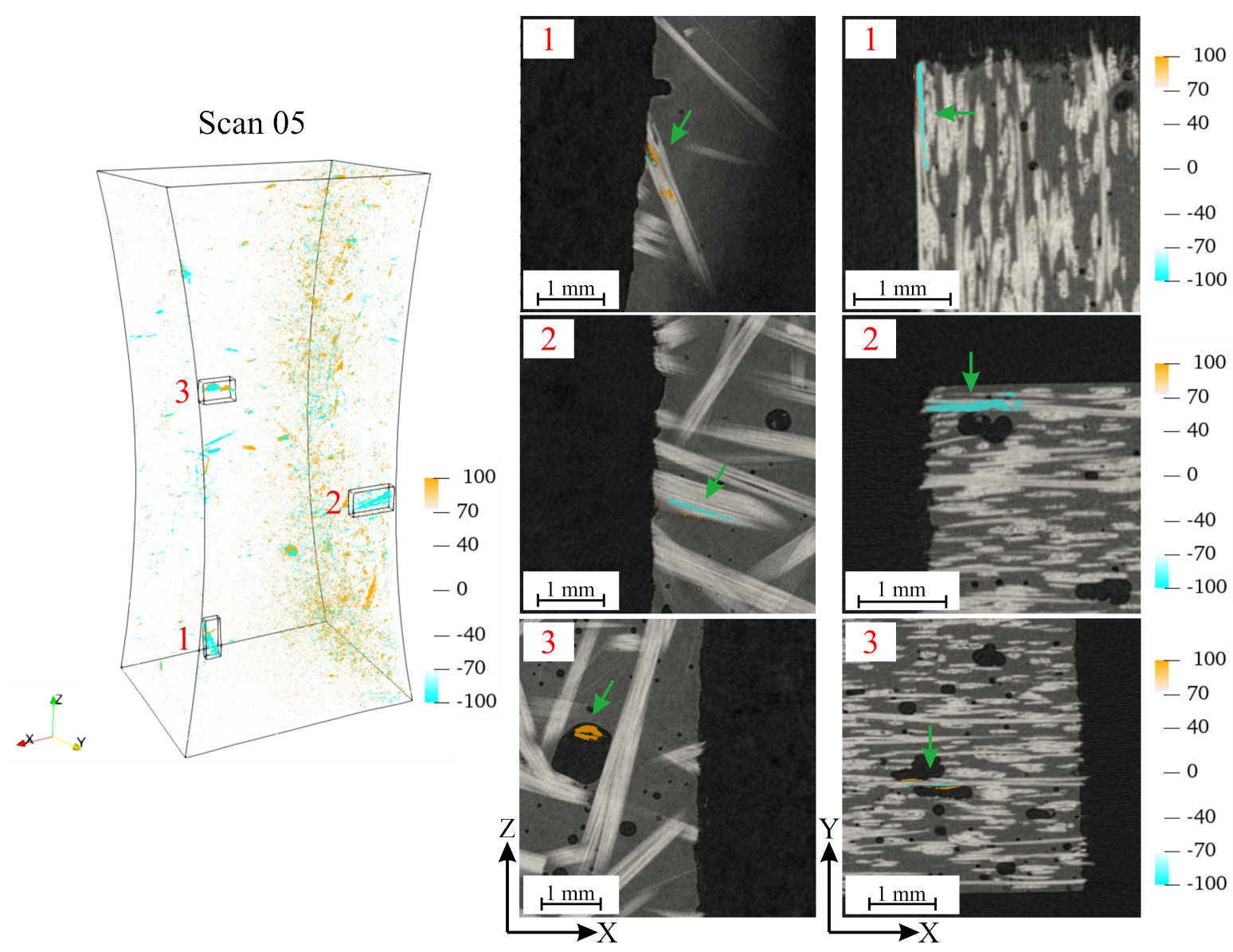

Fig 11. Correlation residuals (scan 05) laid over the corresponding mesostructure sections. The subsets for which comprehensive analyses of correlation residuals and major eigen strain fields are performed are denoted as 1,2 and 3. The green arrows indicate areas of increased correlation residuals in two perpendicular planes. 

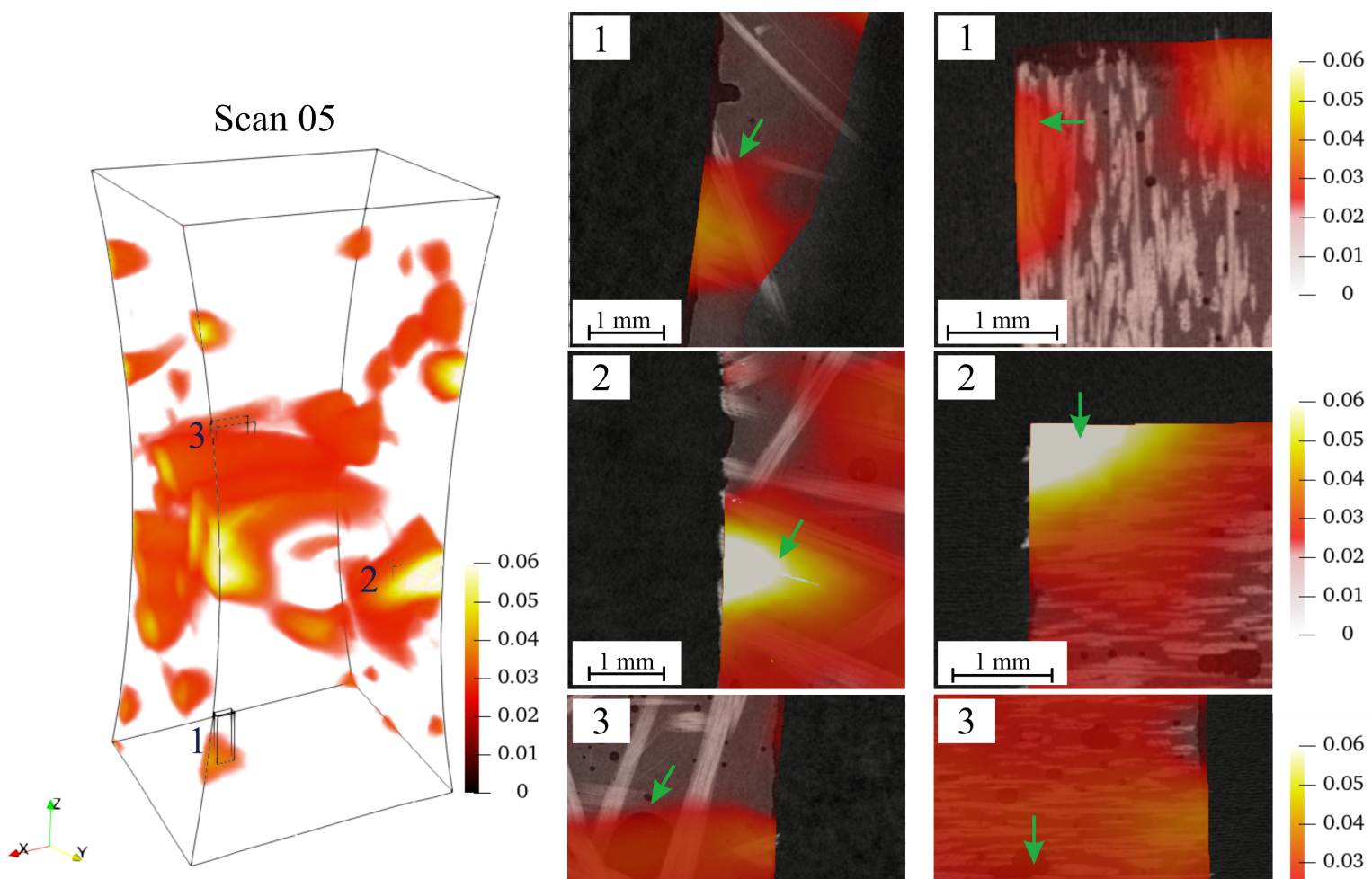

-0.06
-0.05
-0.04
-0.03
-0.02
-0.01
$-\quad 0$
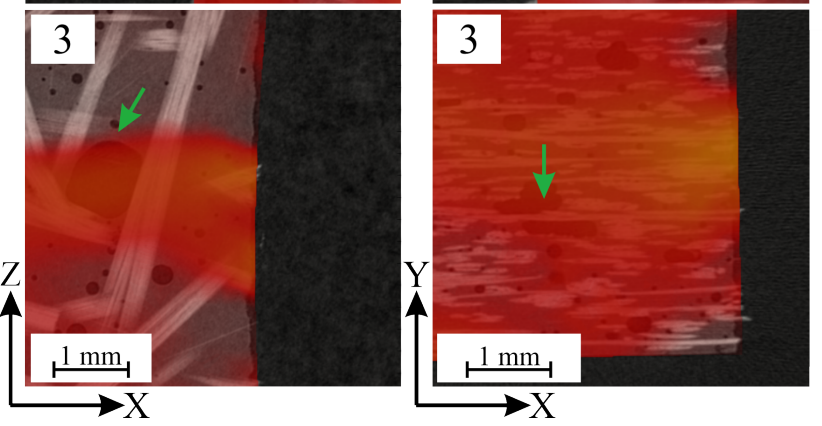

-0.06
-0.05
-0.04
-0.03
-0.02
-0.01
$-\quad 0$

Fig 12. Major eigen strain fields (scan 05) plotted over the corresponding mesostructure sections. Characteristics subsets for which comprehensive analyses of correlation residuals and major eigen strain fields are conducted are denoted with 1,2 and 3 . The green arrows indicate strain localization areas in two perpendicular planes corresponding to regions of increased correlation residuals, as noted in Fig. 11 


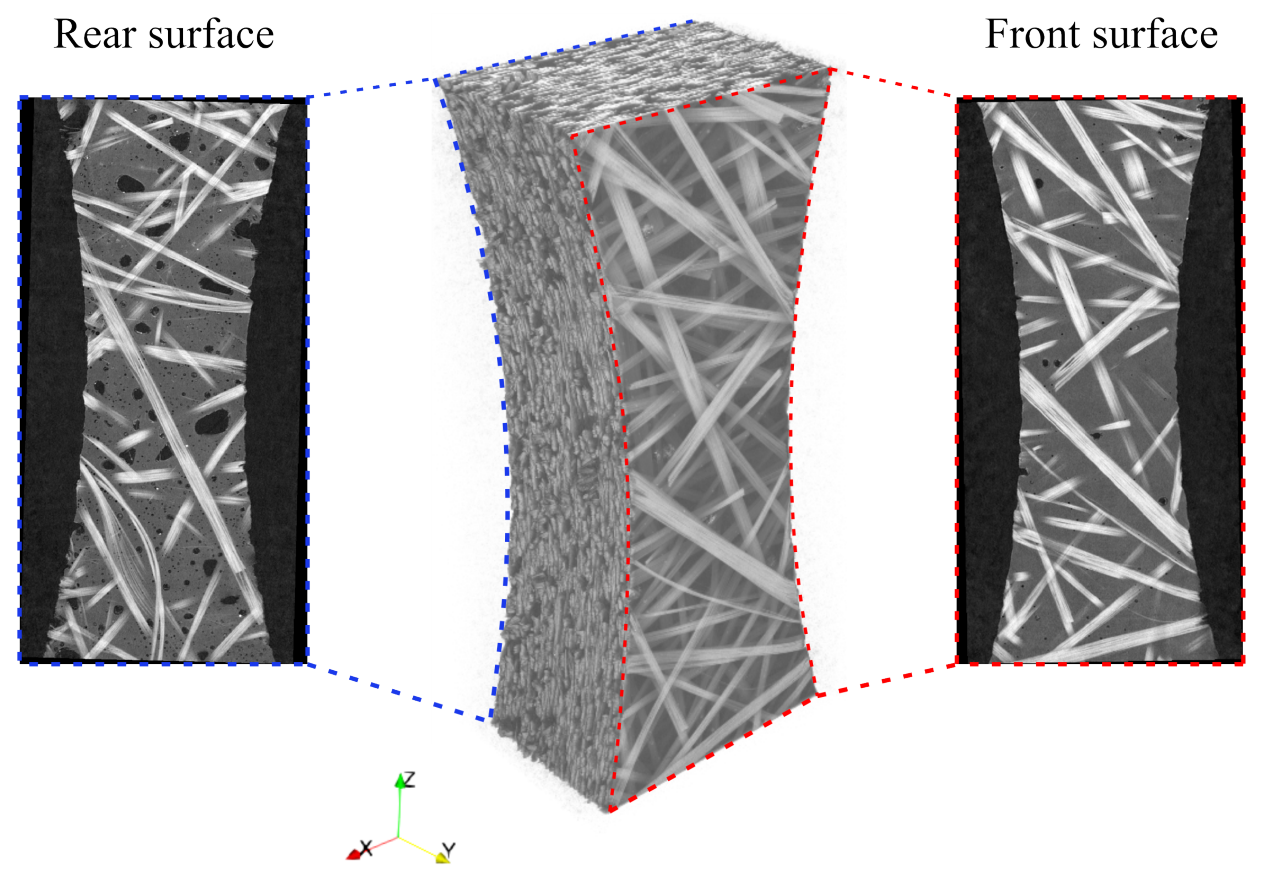

Fig 13. 2D front and rear sections of HQ scan 0 Fig. 3(a) . The corresponding front and rear sections of each acquired scan are then used to perform S-DIC. 

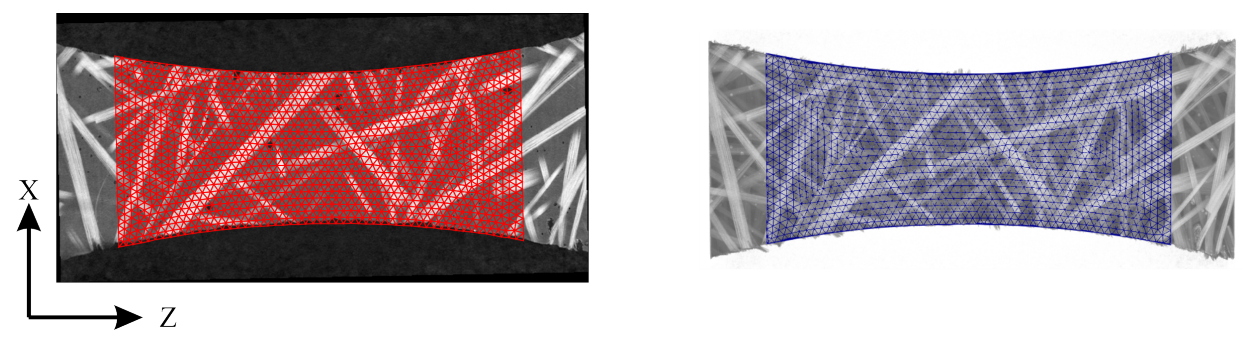

(a)
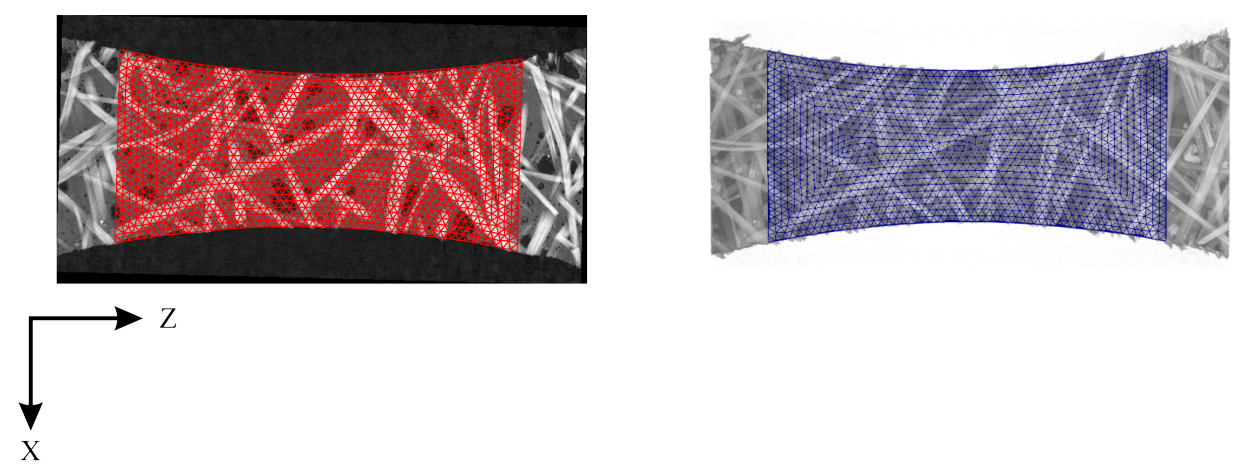

(b)

Fig 14. 2D mesh employed in S-DIC procedure (red) and DVC mesh (blue) plotted over the corresponding mesostructure for the front (a) and rear (b) specimen surfaces. 


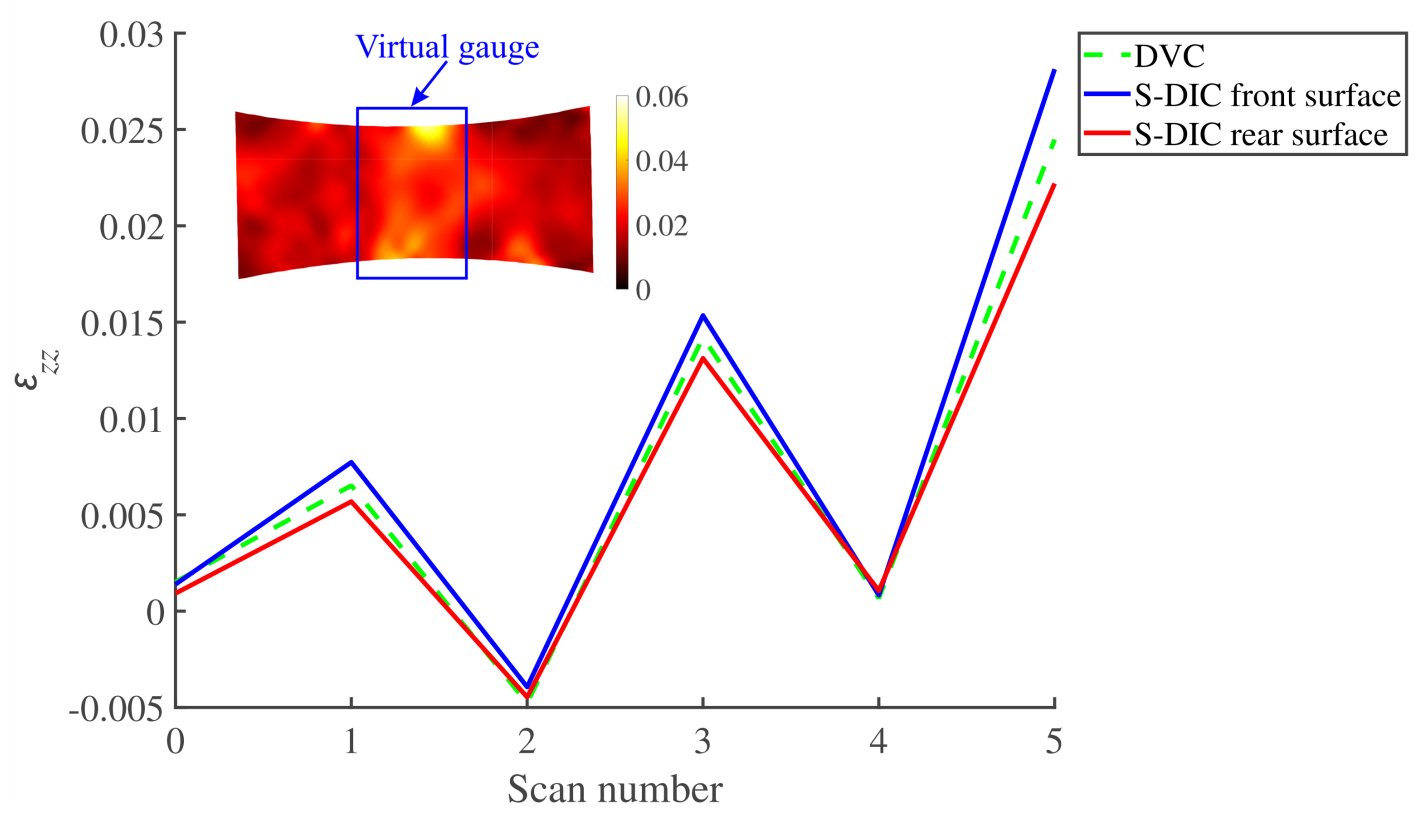

Fig 15. Comparison of mean $\epsilon_{z z}$ strain levels extracted from DVC and S-DIC runs. The mean strain levels are calculated with 2D and 3D virtual gauges of same size positioned over the ROI. The position and size of the gauge are depicted with the blue box on the S-DIC front surface showing $\epsilon_{z z}$ strain field (scan 05). 


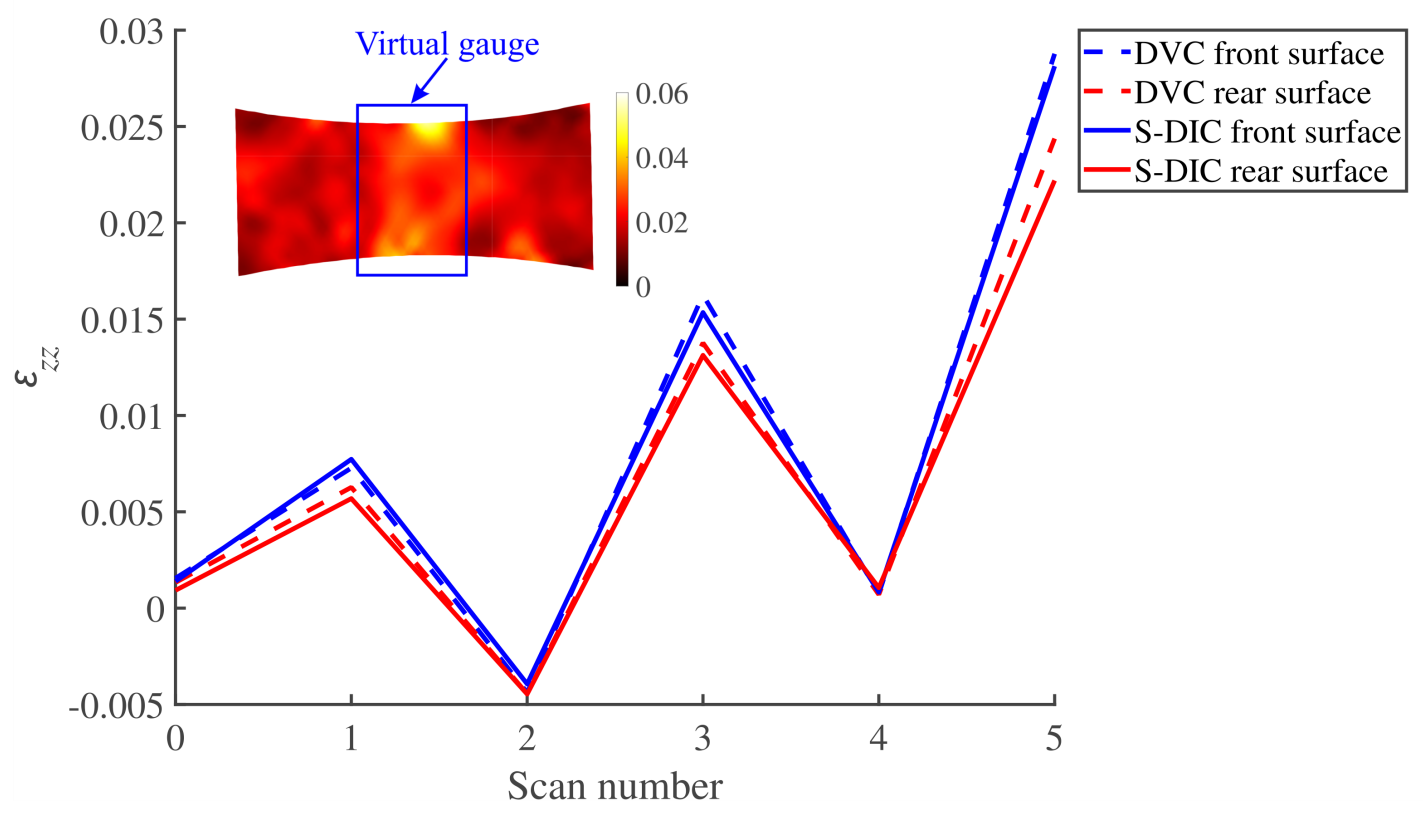

Fig 16. Comparison of mean $\epsilon_{z z}$ strain levels extracted from the front/rear DVC and S-DIC runs. The mean strain levels are calculated via 2D virtual gauges of same size put over the ROI. The gauge position and size are depicted with the blue box in the S-DIC front surface showing $\epsilon_{z z}$ strain field (scan 05). 


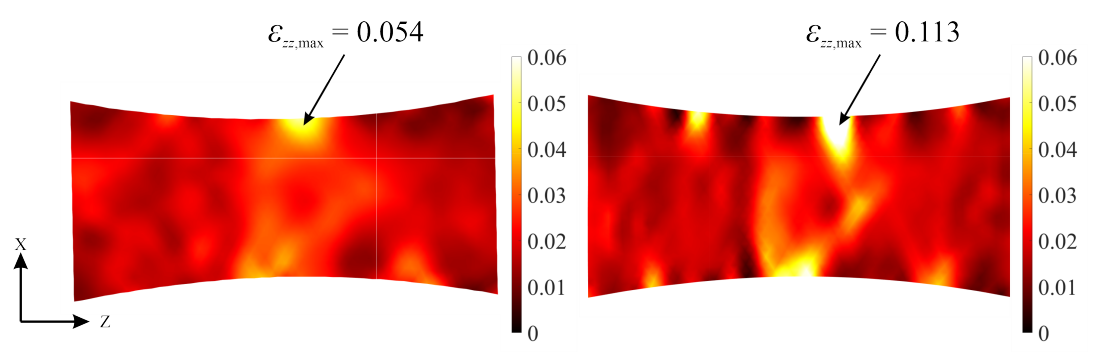

(a)

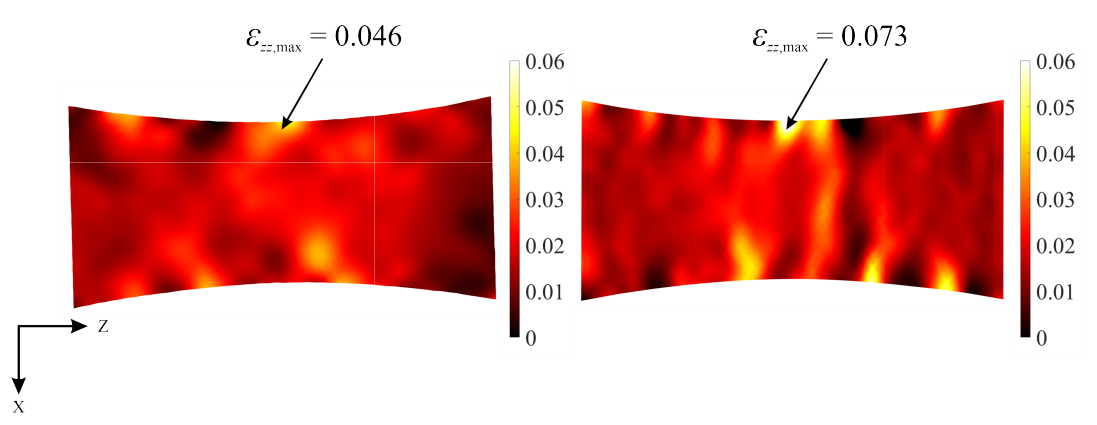

(b)

Fig 17. Comparison of longitudinal strain fields $\varepsilon_{z z}$ obtained with S-DIC (left) and DVC (right) for scan 05 on (a) the front, and (b) rear surfaces. The black arrows depict the location of maximum strain. 

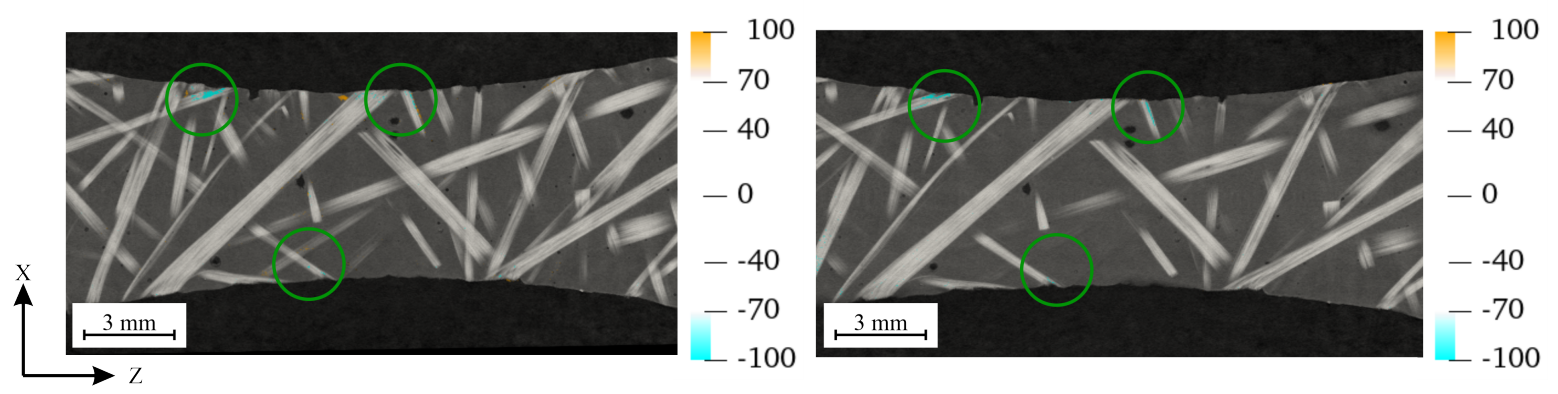

Fig 18. Comparison of correlation residual fields on the front specimen surface obtained with S-DIC (left) and DVC (right) for scan 05. The green circles depict the areas of higher residuals observed on the specimen front surface. 


\section{List of Tables}

$1 \quad$ DIC hardware parameters. . . . . . . . . . . . . . . 51

$2 \quad$ DIC analysis parameters. . . . . . . . . . . . . . . 52

$3 \quad$ Standard displacement and strain uncertainties of DIC measurements. . . . . 53

4 Scanning parameters. . . . . . . . . . . . . . . . . . 54

5 DVC analysis parameters. . . . . . . . . . . . . . . . 55

$6 \quad$ Standard displacement and strain uncertainties of DVC measurements. . . . . 56

$7 \quad$ Stress and maximum eigen strain levels for different load levels of the uniaxial tensile test. . . . . . . . . . . . . . . . . . . 57 
Table 1. DIC hardware parameters.

\begin{tabular}{ll}
\hline Camera & CCD Dalsa \\
\hline Definition & $2358 \times 1728$ pixels \\
Gray levels amplitude & 8 bits \\
Lens & Titanar $50 \mathrm{~mm}$ \\
Aperture & $\mathrm{f} / 2.8$ \\
Field of view & $38 \times 28 \mathrm{~mm}^{2}$ \\
Image scale & $16 \mu \mathrm{m} /$ pixel \\
Stand-off distance & $120 \mathrm{~mm}$ \\
Image acquisition rate & 3 fps \\
Exposure time & $26 \mathrm{~ms}$ \\
Patterning technique & Sprayed black paint \\
\hline
\end{tabular}


Table 2. DIC analysis parameters.

\begin{tabular}{ll}
\hline DIC Software & Correli 3.0 [41] \\
\hline Image filtering & None \\
Element length & $20 \mathrm{px}$ \\
Shape functions & Linear (T3) \\
Mesh & Unstructured Fig. 2(a) \\
Matching criterion & Penalized sum of squared differences \\
Regularization length & Bulk: 10 px, Edges: 10 px \\
Interpolant & Cubic \\
\hline
\end{tabular}


Table 3. Standard displacement and strain uncertainties of DIC measurements.

Displacement noise floor $(x y) \quad 0.012 \mathrm{px} \quad 0.012 \mathrm{px}$

Strain $\varepsilon_{1}$ noise floor $\quad 8 \times 10^{-5} \mathrm{px}$


Table 4. Scanning parameters.

\begin{tabular}{|c|c|c|}
\hline Tomograph & \multicolumn{2}{|l|}{ North Star Imaging X50+ } \\
\hline $\mathrm{X}$-ray source & \multicolumn{2}{|l|}{ XRayWorX XWT-240-CT } \\
\hline Target/ Anode & \multicolumn{2}{|l|}{ W (reflection mode) none } \\
\hline Filter & \multicolumn{2}{|l|}{ None } \\
\hline Voltage & \multicolumn{2}{|l|}{$180 \mathrm{kV}$ (actual $149.661 \mathrm{kV}$ ) } \\
\hline Current & \multicolumn{2}{|l|}{$50 \mu \mathrm{A}$ (actual 77.642) } \\
\hline Focal spot size & \multicolumn{2}{|l|}{$5 \mu \mathrm{m}$} \\
\hline Tube to detector & \multicolumn{2}{|l|}{$910 \mathrm{~mm}$} \\
\hline Tube to object & \multicolumn{2}{|l|}{$53 \mathrm{~mm}$} \\
\hline Detector & \multicolumn{2}{|l|}{ Dexela 2923} \\
\hline Definition & \multicolumn{2}{|c|}{$1507 \times 1849$ pixels $(2 \times 2$ binning $)$} \\
\hline Scanning parameters & High quality (HQ) & Low quality (LQ) \\
\hline Number of projections & 800 & 768 \\
\hline Angular amplitude & $360^{\circ}$ & $360^{\circ}$ \\
\hline Frame average & 20 per projection & Continuous ( 1 per average) \\
\hline Frame rate & $3 \mathrm{fps}$ & $3 \mathrm{fps}$ \\
\hline Acquisition duration & $1 \mathrm{~h} 46 \min 26 \mathrm{~s}$ & $4 \min 26 s$ \\
\hline Reconstruction algorithm & filtered back-projection & filtered back-projection \\
\hline Gray levels amplitude & 8 bits & 8 bits \\
\hline Volume size & $499 \times 685 \times 1401$ (after crop) & $499 \times 685 \times 1401$ (after crop) \\
\hline Field of view & $7.3 \times 10 \times 20.5 \mathrm{~mm}^{3}$ & $7.3 \times 10 \times 20.5 \mathrm{~mm}^{3}$ \\
\hline Image scale & $14.6 \mu \mathrm{m} /$ voxel & $14.6 \mu \mathrm{m} /$ voxel \\
\hline Pattern & Natural (Fig. 4) & Natural (Fig. 4) \\
\hline
\end{tabular}


Table 5. DVC analysis parameters.

\begin{tabular}{ll}
\hline DVC software & Correli 3.0 [41] \\
\hline Image filtering & None \\
Element length (mean) & 18 voxels \\
Shape functions & Linear (T4) \\
Mesh & Unstructured Fig. 2(b) \\
Matching criterion & Penalized sum of square differences \\
Regularization length & Bulk: 96 voxels, Dirichlet surfaces: 64 voxels \\
Interpolant & Cubic \\
\hline
\end{tabular}


Table 6. Standard displacement and strain uncertainties of DVC measurements.

\begin{tabular}{llll}
\hline Displacement noise floor $(x y z)$ & $0.083 \mathrm{vx}$ & $0.061 \mathrm{vx}$ & $0.036 \mathrm{vx}$ \\
Strain noise floor $\left(\varepsilon_{1}\right)$ & \multicolumn{3}{c}{$8 \times 10^{-5} \mathrm{vx}$} \\
\hline
\end{tabular}


Table 7. Stress and maximum eigen strain levels for different load levels of the uniaxial tensile test.

\begin{tabular}{ccc}
\hline Image number & $\sigma, \mathrm{MPa}$ & $\varepsilon_{1}$ \\
\hline 40 & - & - \\
100 & 37 & 0.006 \\
150 & 80 & 0.013 \\
195 & 119 & 0.018 \\
265 & 164 & 0.026 \\
299 & 178 & 0.037 \\
\hline
\end{tabular}

